\title{
Educação física escolar: Guia de Atividade Física para a População Brasileira
}

\section{Physical Education: Physical Activity Guidelines for the Brazilian Population}

\section{AUTORES \\ Kelly Samara da Silva ${ }^{1}$ (D) \\ Alexsandra da Silva Bandeira ${ }^{1}$ (D) \\ Fabricio Cesar de Paula Ravagnani ${ }^{2}$ (D) \\ Edina Maria de Camargo ${ }^{3}$ (i) \\ Maria Cecília Tenório ${ }^{4}$ (D) \\ Victor José Machado de Oliveira 5 (iD) \\ Priscila Cristina dos $\operatorname{Santos}^{1}$ (D) \\ Virgílio Viana Ramires ${ }^{6}$ (D) \\ Paula Fabricio Sandreschi ${ }^{1,7}$ (ID) \\ Pedro Curi Hallal ${ }^{8}$ (D) \\ Valter Cordeiro Barbosa Filho 9,10 (iD \\ 1 Universidade Federal de Santa Catarina, Departamento de Educação Física, Florianópolis, Santa Catarina, Brasil. \\ 2 Instituto de Educação Ciência e Tecnologia de Campo Grande, Mato Grosso do Sul, Brasil \\ 3 Universidade Tecnológica Federal do Paraná, Depar- tamento de Educação Fisica, Curitiba, Paraná, Brasil. \\ 4 Universidade Federal Rural de Pernambuco, Departamento de Educação Física, Recife, \\ Pernambuco, Brasil. \\ 5 Universidade Federal do Amazonas, Faculdade de Educação Física e Fisioterapia, Programa de Pós-Graduação em Educação, Manaus, Amazonas, \\ 6 Instituto Federal de Educação, Ciência e Tecnologia Sul-rio-grandense - Câmpus Camaquã Camaqua, Rio Grande do Sul, Brasil. \\ 7 Ministério da Saúde, Secretaria de Atenção Primária à Saúde, Departamento de Promoção da Saude, Coordenaçao-Geral de Promoçao da Distrito Federal, Brasil. \\ 8 Universidade Federal de Pelotas, Departamento de Ginástica e Saúde, Pelotas, Rio Grande do Sul, Brasil. 9 Instituto de Educação, Ciência e Tecnologia do Ceará - Campus Aracatí, Aracati, Ceará, Brasil. 10 Universidade Estadual do Ceará, Programa de Pós-Graduação em Saúde Coletiva, Fortaleza, Ceará, Brasil.}

\section{CONTATO}

Kelly Samara da Silva

ksilvajp@gmail.com

Universidade Federal de Santa Catarina, Centro de Desportos - Campus Trindade. Av. César Seara. Bloco 3, Florianópolis, Santa Catarina.

CEP: 88040-900.

DOI

10.12820/rbafs.26e0219

\section{(cc) BY}

Este trabalho está licenciado com uma Licença Creative Commons - Atribuição 4.0 Internacional.

\section{RESUMO}

As aulas de educação física (EF) escolar representam um momento propício para conhecer e vivenciar a pluralidade do movimento humano, favorecendo a promoção de uma vida ativa e saudável. A importância da EF escolar foi reconhecida pelo Guia de Atividade Física para a População Brasileira (Guia), que dedicou um capítulo para orientar a comunidade escolar sobre os benefícios à saúde e as estratégias de sucesso adotadas nas aulas de EF escolar. O objetivo deste estudo é apresentar o processo de elaboração das recomendações brasileiras de EF escolar para a população brasileira, mais especificamente os estudantes, professores, pais e responsáveis, e gestores. Esse capítulo do Guia foi desenvolvido por dez pesquisadores brasileiros, incluindo um representante do Ministério da Saúde. Três sínteses de evidências, escutas ao público-alvo e consulta pública foram realizadas para a construção das recomendações. Dados de 49 revisões sistemáticas sobre os benefícios da EF escolar à saúde e de 22 intervenções nas aulas de EF escolar com estudantes brasileiros foram sumarizados. Ainda, foram sintetizados 63 documentos nacionais e internacionais com estratégias recomendadas para a EF escolar, com foco nas dimensões: política e ambiente; currículo; instrução apropriada; avaliação; e estratégias que interagem com a EF escolar. Os grupos focais e a consulta pública fundamentaram a elaboração e o aprimoramento da versão final das recomendações. Espera-se que estas recomendações alicercem a criação de políticas públicas, melhorem a conscientização sobre a importância da prática de atividade física na EF escolar e enfatizem a necessidade de realizar estudos futuros nesta área de conhecimento.

Palavras-chave: Educação física; Atividade motora; Estratégias; Escola; Estudantes.

\section{ABSTRACT}

Physical Education (PE) classes represent an appropriate time to know and experience the plurality of the human movement, favoring the promotion of a healthy and active life. The importance of PE was recognized by the Physical Activity Guide for the Brazilian Population, which dedicated a chapter to guide the school community on the health benefits and successful strategies adopted in PE classes. This study aimed to show the process of elaborating Brazilian recommendations for PE for the Brazilian population, more specifically students, teachers, parents and guardians, and managers. This chapter of the Guide was developed by ten Brazilian researchers, including a spokeswoman of the Ministry of Health. Three syntheses of evidence, focus groups/interviews and public consultation were carried out to construct the recommendations. Data from 49 systematic reviews on the health benefits of PE and 22 interventions in PE classes with Brazilian students were summarized. Also, 63 documents were synthesized with strategies recommended for PE classes, focusing on the dimensions: politics and environment; curriculum; appropriate instruction; evaluation; and strategies that interact with PE. The focus groups and the public consultation were the basis for the elaboration and improvement of the final version of the recommendations. It is expected that these recommendations can assist the creation of public policies, the improvement of practice PE and the need to conduct future studies in this area of knowledge.

Keywords: Physical education; Motor activity; Strategies; School; Students. 


\section{Introdução}

A educação física (EF) escolar é um componente curricular obrigatório da educação básica no Brasil ${ }^{1}$, referenciada na Base Nacional Comum Curricular ${ }^{2}$. A natureza híbrida da EF escolar integra aspectos da educação e da saúde, por meio de conhecimento, aprendizado e experiências corporais que contribuem para uma ou mais dimensões da saúde (física e motora; psicológica; social e ambiental; e cognitiva $)^{3-11}$. Com isso, inúmeras estratégias têm sido avaliadas e implementadas (relativas a dimensões como política e ambiente; currículo; instruções apropriadas; e avaliação dos estudantes) $)^{12}$,com o intuito de impactar positivamente na vida e na saúde dos estudantes ${ }^{3-11}$.

A contribuição positiva das aulas de EF escolar para a saúde ${ }^{3-11}$ tem guiado a construção de recomendações voltadas para a EF escolar em diferentes países ${ }^{12-17}$. Benefícios têm sido reportados em indicadores da saúde física e motora, como aptidão cardiorrespiratória $(\mathrm{ACR})^{18}$ e habilidades motoras fundamentais ${ }^{18}$; da saúde psicológica, como engajamento, motivação e autonomia ${ }^{7}$, afetividade ${ }^{6,9}$, redução da ansiedade e depressão $0^{5}$ e da saúde socioambiental, com ênfase na empatia e cooperação $0^{5,19,20}$, fazer amigos ${ }^{7,20}$ e comportamento pró-social ${ }^{19,20}$. Revisões recentes têm também mostrado os benefícios das aulas de EF escolar no desempenho escolar (melhora na atenção, concentração, memória, comportamento em aula, solução de problemas e média de notas) ${ }^{21,22}$ e na saúde cognitiva (tomada de decisão, habilidades técnicas e orientação da tarefa) ${ }^{23,24}$.

As estratégias utilizadas para melhorar a saúde dos escolares por meio das aulas de EF escolar são diversas e se organizam em dimensões específicas como política e ambiente (exemplo, valorização e estrutura), instrução apropriada (exemplo, utilização de recursos nas aulas e promoção da prática de atividade física), currículo (exemplo, progressão de conteúdos e uso de diretrizes educacionais) e avaliação (exemplo, desenvolvimento e progressão de diferentes habilidades) ${ }^{12}$. Além disso, há estratégias que não ocorrem especificamente na $\mathrm{EF}$ escolar, mas interagem com a disciplina, como por exemplo, oferta de esportes ou atividade física no contraturno. No Brasil, programas governamentais apoiam essas estratégias no ambiente escolar, como o Programa Saúde na Escola ${ }^{25}$ e o Programa Segundo Tempo ${ }^{26}$.

O presente estudo sistematiza o processo de construção do Capítulo 6 "Educação Física Escolar", elaborado para o Guia de Atividade Física para a População Brasileira (Guia). Informações detalhadas deste
Guia podem ser consultadas em publicações prévias ${ }^{27}$. O propósito deste trabalho foi sintetizar o processo de construção desta seção do Guia, com ênfase na descrição dos aspectos metodológicos e das principais evidências que nortearam as recomendações de aulas de EF escolar para o Brasil.

\section{Método}

O grupo de trabalho se reunia uma vez por semana, no período de maio de 2020 a janeiro de 2021, utilizando recursos da plataforma do Google Meet. Todas as decisões foram registradas e compartilhadas em pastas no Dropbox. O planejamento, a execução das atividades e o cronograma de trabalho foram definidos nos primeiros encontros, estabelecendo-se a execução de: (i) revisões sistemáticas; (ii) escuta ao público-alvo das recomendações; (iii) consulta pública, conforme descrito na Figura $1^{27}$.

A primeira etapa do processo de construção da recomendação foi a condução de três revisões de escopo, as quais foram realizadas com o intuito de fundamentar as evidências científicas relevantes para a construção do documento. Para mapear a contribuição das aulas de EF escolar para a saúde, uma síntese de revisões sistemáticas sobre intervenções na EF escolar foi realizada. Em segui$\mathrm{da}$, foram sumarizados estudos brasileiros de intervenção na EF escolar que visavam mudanças na saúde dos estudantes. Por fim, foram mapeadas as principais estratégias recomendadas para as aulas de EF escolar com foco na promoção de um estilo de vida ativo e saudável.

As revisões foram realizadas para obter uma visão geral de pesquisas disponíveis sobre o tema para registro de suas principais características, evidências e lacunas dos estudos ${ }^{28}$. Essas revisões foram desenvolvidas seguindo protocolos internacionais de sínteses de estudos $^{28}$, considerando as seguintes etapas: 1 ) identificação do problema de pesquisa; 2) identificação dos estudos relevantes; 3) seleção dos estudos; 4) extração dos dados; e 5) sumarização dos resultados. Foram considerados documentos publicados a partir do ano 2000, nas línguas portuguesa, inglesa e espanhola. Informações detalhadas dos elementos e critérios utilizados são encontrados em publicação prévia ${ }^{27}$.

As revisões foram estruturadas com base nas seguintes perguntas: (i) $\mathrm{O}$ que as sínteses de evidências relatam sobre a contribuição das aulas de EF escolar para diferentes componentes do estilo de vida ativo e saudável em estudantes? (ii) Quais as evidências de contribuições das intervenções implementadas nas aulas de EF escolar no Brasil para promoção de um estilo de vida ativo e 


\section{RECOMENDAÇÕES PARA AS AULAS DE EDUCAÇÃO FÍSICA ESCOLAR - BRASIL}

GRUPO DE TRABALHO - EF ESCOLAR

REUNIÕES SEMANAIS (julho 2020 até janeiro 2021)

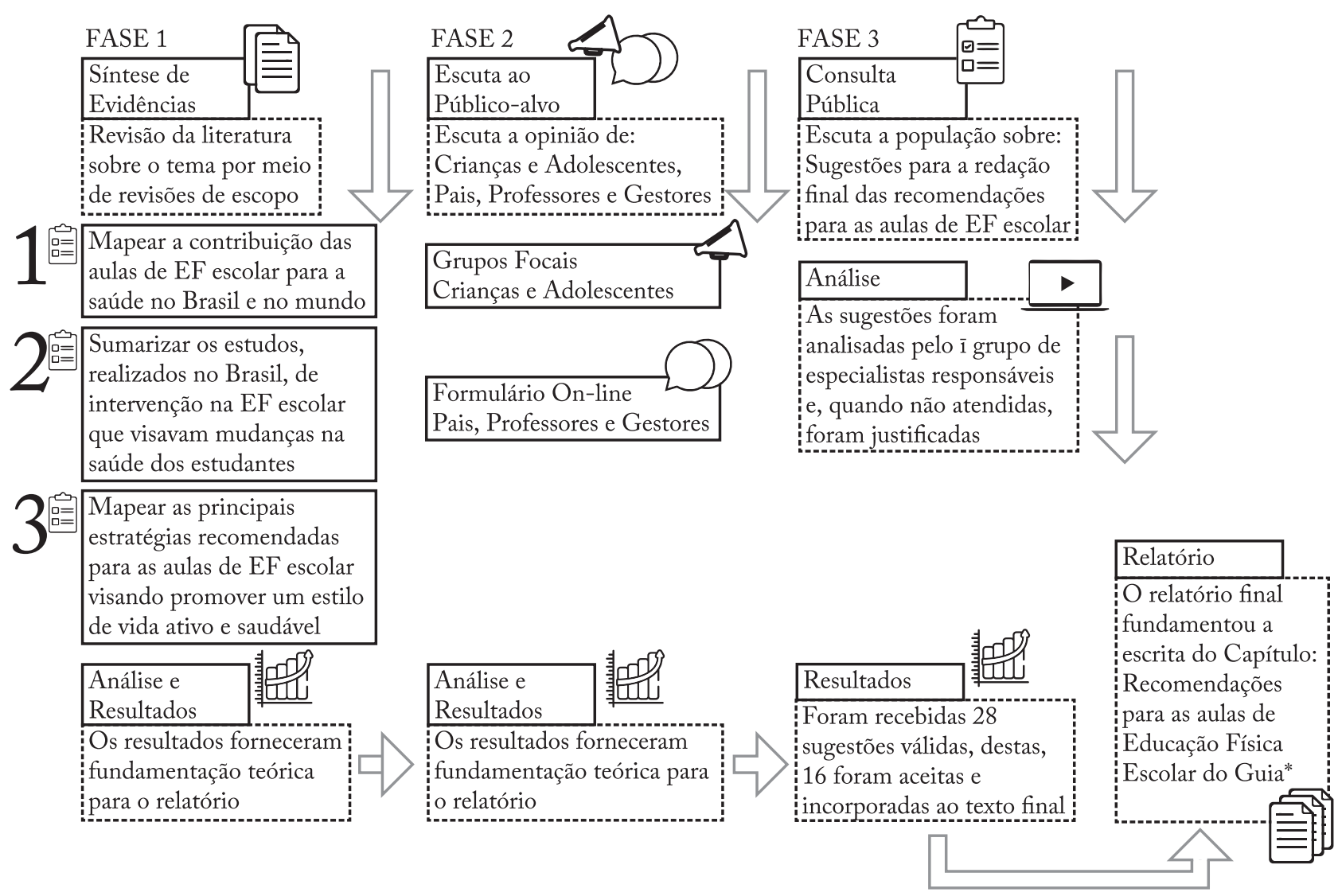

Figura 1 - Descrição das fases de elaboração das Recomendações para as aulas de Educação Física escolar.

saudável? e (iii) Quais as estratégias relacionadas às aulas de EF escolar têm sido recomendadas para promoção de um estilo de vida ativo e saudável em estudantes?

No dia 04 de junho de 2020 as bases de dados/repositórios eletrônicos WEB OF SCIENCE, MEDLINE/ PUBMED, LILACS, SCIELO, ERIC Proquest, PsycINFO, Scopus e SPORTDiscus foram acessadas. No mesmo mês, 82 (oitenta e dois) portais de instituições nacionais e internacionais de EF escolar; atividade física; esporte; saúde ou educação foram consultados. Além disso, procedeu-se com busca secundária por estudos em seções de referências de duas revisões de revisões ${ }^{29,30} \mathrm{e}$ na biblioteca pessoal dos pesquisadores envolvidos no projeto $^{27}$. Os títulos alcançados foram importados para uma biblioteca do software EndnoteWeb. As referências duplicadas foram excluídas utilizando as funções do gerenciador de referências e manualmente. Os títulos elegíveis foram organizados e analisados separadamente por quatro avaliadores (ASB, PCS, FR e VR). A eta- pa de seleção envolveu: 1) leitura dos títulos e resumos; 2) leitura dos documentos na íntegra. Em caso de discordâncias entre os avaliadores, um terceiro avaliador (VBF) foi consultado para o consenso. A consulta aos sites foi realizada por pelo menos um dos avaliadores (ASB, PCS, VBF; MCMT e PFS) e a análise dos documentos elegíveis foi feita por quatro avaliadores (ASB, PCS, FR e VR).

A extração dos dados foi realizada por dois pesquisadores, utilizando instrumento padronizado. Na questão 1 , as principais evidências dos estudos foram agrupadas e discutidas. Revisões que adotaram metanálise ou estratégias para avaliar o grau de certeza das evidências foram enfatizadas. $\mathrm{Na}$ questão 2, as intervenções no Brasil foram descritas considerando o contexto de implementação, as estratégias utilizadas, os desfechos e os principais resultados. $\mathrm{Na}$ questão 3, as recomendações foram organizadas nas dimensões que contemplam os componentes essenciais da educação física (política e 
meio ambiente, currículo, instrução apropriada, avaliação ou estratégias que integram com a EF).

A segunda etapa da construção das recomendações tratou de ouvir os atores (estudantes, professores, pais/ responsáveis e gestores da educação) envolvidos nesta pesquisa, para acessar informações importantes à elaboração das recomendações voltadas à EF escolar. Essas informações foram obtidas por meio de técnica de grupos focais para os estudantes e do preenchimento de formulários on-line para professores, pais/responsáveis e gestores.

Os participantes foram selecionados por conveniência, com idades de 10 a 13 anos $(60 \%$ meninas; $\mathrm{n}=9)$ e de 14 a 17 anos (60\% meninas, $n=15)$ matriculados na rede pública de ensino de diferentes regiões do país. As sessões foram feitas de forma remota, com a moderação dos grupos realizada por um único pesquisador. Os grupos focais, com duração média de 60 minutos, seguiram uma matriz conceitual com roteiro semiestruturado dividido em três partes: fatores intrapessoal, interpessoal e ambiental para prática de atividade física nas aulas de $\mathrm{EF}$ escolar ${ }^{27}$. As respostas e discussões foram registradas e gravadas pelo sistema remoto do Google Meet. Os arquivos foram transcritos na íntegra, e as frases e informações obtidas foram agrupadas de acordo com seus temas e analisadas por meio de abordagem qualitativa com o emprego da análise de conteúdo.

Perguntas para professores de EF escolar (12 questões), pais/responsáveis (16 questões) e gestores estaduais e municipais (16 questões) foram elaboradas e disponibilizadas no Google Forms. As questões abertas e fechadas sobre a importância, as barreiras e os facilitadores para as aulas de EF escolar foram elaboradas com base nas revisões de literatura descritas anteriormente. A amostra foi selecionada por conveniência. Os formulários foram enviados por e-mail ou aplicativo de troca de mensagens, e a participação foi voluntária, com garantia do anonimato dos respondentes ${ }^{27}$.

Após a redação da primeira versão do Guia de Atividade Física para População Brasileira, o documento foi aberto à consulta pública em agosto de 2020. O texto foi hospedado no website do Ministério da Saúde, bem como um formulário online onde a população geral poderia registrar sugestões para a redação final do Guia. As sugestões foram analisadas pelo grupo de especialistas responsáveis pela elaboração das recomendações e, quando não atendidas, foram justificadas ${ }^{27}$. Para o capítulo referente a EF escolar, foram recebidas 28 sugestões válidas, destas, 16 foram aceitas e incorporadas ao texto final por possuírem relevância técnica e/ou social.

\section{Resultados}

Para responder à pergunta "O que as sinteses de evidências relatam sobre as contribuiçôes das aulas de EF escolar para diferentes componentes do estilo de vida ativo e saudável em estudantes?", foram analisadas 2.287 referências recuperadas das bases de dados, repositórios, home-pages e listas de referências. Destas, 2.136 foram excluídas por screening dos títulos e resumos, restando 107 artigos e 46 documentos para leitura na íntegra. Após a leitura completa dos artigos e documentos, foram incluídas 45 revisões sistemáticas e quatro documentos institucionais (lista de referências no material suplementar 1). Os desfechos foram agrupados em dimensões (atividade física; saúde física e motora; psicológica; socioambiental e cognitiva) e os resultados apresentados na Tabela 1 . Das 49 revisões analisadas, 23 avaliaram se intervenções nas aulas de EF escolar influenciaram a prática de atividade física. Em relação a saúde física e motora, 22 revisões avaliaram o impacto da EF com evidências mais consistentes para melhora na aptidão cardiorrespiratória, habilidades motoras fundamentais, flexibilidade e índice de massa corporal. Quanto à saúde psicológica dos estudantes, observou-se que 9 revisões analisaram a relação da EF com essa dimensão da saúde e todas destacaram resultados positivos. Somente quatro revisões analisaram o efeito da EF sobre desfechos de saúde socioambiental e 13 revisões analisaram aspectos cognitivos. As principais estratégias mencionadas para aumentar a prática de atividade física nas aulas de EF escolar foram: aumento do tempo e da frequência das aulas em onze revisões, aumento na intensidade das aulas em sete revisões, mudanças no conteúdo em oito revisões e na qualidade das aulas em cinco revisões. Maiores informações serão detalhadas em publicações futuras (in press).

Para a segunda pergunta, "Quais as evidências de contribuições das intervençôes implementadas nas aulas de EF escolar no Brasil para promoção de um estilo de vida ativo e saudável?" Foram encontrados 896 documentos potencialmente elegíveis na literatura e, após exclusão das duplicatas, ficaram 737. Um total de 35 documentos foram incluídos nesta revisão (lista de referências no material suplementar 2), referentes a 22 estudos de intervenção. Desses, 16 apresentaram resultados de efetividade de que intervir nas aulas de EF escolar contribui para a melhoria de algum indicador de saúde, incluindo aumento do tempo em atividade física observado em cinco estudos; redução no tempo de tela e melhoria dos hábitos alimentares em quatro estudos; melhoria em indicado- 
Tabela 1 - Síntese dos principais efeitos das intervenções realizadas nas aulas de educação física (EF) escolar sobre diferentes dimensões da saúde em estudantes de diferentes faixas etárias

\begin{tabular}{|c|c|c|c|c|c|c|c|}
\hline \multicolumn{2}{|c|}{ Identificação do estudo } & \multicolumn{6}{|c|}{ Indicador de saúde } \\
\hline Referências & $\begin{array}{l}\text { Ano escolar/idade } \\
\left(\mathrm{EF}^{¥} \text { ou } \mathrm{EM}^{¥ ¥}\right)\end{array}$ & $\mathrm{AF \#}$ & $\begin{array}{c}\text { Outros } \\
\text { comportamentos }\end{array}$ & $\begin{array}{c}\text { Saúde física/ } \\
\text { motora }\end{array}$ & $\begin{array}{c}\text { Saúde } \\
\text { psicológica }\end{array}$ & $\begin{array}{c}\text { Saúde } \\
\text { socioambiental } \\
\end{array}$ & $\begin{array}{c}\text { Saúde cognitiva/ } \\
\text { desempenho escolar }\end{array}$ \\
\hline \multicolumn{8}{|l|}{ Revisões sistemáticas } \\
\hline Álvarez-Bueno et al. ${ }^{1}$ & $1^{\circ} \mathrm{EF}$ ao $3^{\circ} \mathrm{EM}$ & & & & & & $\uparrow$ \\
\hline Barba-Martín et al.2 & NR & & & & $?$ & & \\
\hline Becerra-Fernández et al. ${ }^{3}$ & $1^{\circ}$ ao $3^{\circ} \mathrm{EM}$ & & & $\uparrow$ & & & \\
\hline Becerra Fernandez et al. ${ }^{4}$ & Até 11 anos & & & $\uparrow$ & & & \\
\hline Bessa et $\mathrm{al}^{5}{ }^{5}$ & NR & & & & $\uparrow$ & $\uparrow$ & \\
\hline Braithwaite et al. ${ }^{6}$ & NR & & & $\uparrow$ & $\uparrow$ & & $\uparrow$ \\
\hline Camacho-Miñano et al. ${ }^{7}$ & Ensino primário até o EM & $\uparrow$ & & & & & \\
\hline Chacón-Borrego et al. ${ }^{8}$ & Ensino primário até o EM & & & $\uparrow$ & & & \\
\hline Dobbins et al. ${ }^{9}$ & 6 até 18 anos & & & $\uparrow$ & & & \\
\hline Donnelly et al. ${ }^{10}$ & $\mathrm{EF}(5-13$ anos $)$ & & & & & & $?$ \\
\hline Dudley et al. ${ }^{11}$ & 3 até 18 anos & $\uparrow$ & & $\uparrow$ & & & $\uparrow$ \\
\hline Dudley et al. ${ }^{12}$ & 5 até 18 anos & & & $\uparrow$ & $\uparrow$ & & \\
\hline Gomes et al. ${ }^{13}$ & 6 até 10 anos & & & & $\uparrow$ & & \\
\hline Gunnell et al. ${ }^{14}$ & Até 18 anos. & & & & & & $?$ \\
\hline Hermoso et al. ${ }^{15}$ & 3 até 18 anos & & & $\uparrow$ & & & \\
\hline Hoehner et al. ${ }^{16}$ & NR & $\uparrow$ & & & & & \\
\hline Jenkinson et al. ${ }^{17}$ & 5 até 18 anos & $?$ & & $?$ & & & \\
\hline Kahn et al. ${ }^{18}$ & 5 até 17 anos & $\uparrow$ & & $\uparrow$ & & & \\
\hline Kopp et al. ${ }^{19}$ & 6 até 18 anos & $\uparrow$ & & & & & \\
\hline Lai et al..$^{20}$ & 3 até 18 anos & $\uparrow$ & & $\uparrow$ & & & \\
\hline Lambrinou et al..$^{21}$ & Somente crianças & $\uparrow$ & $\uparrow$ & $\uparrow$ & & & \\
\hline Lonsdale et al. ${ }^{22}$ & $1^{\circ} \mathrm{EF}$ ao $3^{\circ} \mathrm{EM}$ & $\uparrow$ & & & & & \\
\hline McDonald et al..$^{23}$ & 3 até 18 anos & $\uparrow$ & & & & & \\
\hline Marques et al. ${ }^{24}$ & 3 até 18 anos & & & & & & $\uparrow$ \\
\hline Matson-Koffmannet al. ${ }^{25}$ & EF e EM & $\uparrow$ & $\uparrow$ & & & & \\
\hline Minattoet al. ${ }^{26}$ & 10 até 19 anos & & & $\uparrow$ & & & \\
\hline Morgan et al. ${ }^{27}$ & Ensino primário até o EM & & & $\uparrow$ & & & \\
\hline Opstoel et al. ${ }^{28}$ & 6 até 18 anos & & & & & $\uparrow$ & \\
\hline Quitério et al. ${ }^{29}$ & $1^{\circ} \mathrm{EF}$ ao $3^{\circ} \mathrm{EM}$ & $\uparrow$ & $\uparrow$ & $\uparrow$ & & & \\
\hline Rasberry et al. ${ }^{30}$ & 5 até 18 anos & & & & & & $\uparrow$ \\
\hline Robinson et al. ${ }^{31}$ & $1^{\circ} \mathrm{EF}$ ao $3^{\circ} \mathrm{EM}$ & $\uparrow$ & & $\uparrow$ & & & \\
\hline Salmon et al. ${ }^{32}$ & 4 até 19 anos & $\uparrow$ & & & & & \\
\hline Sharma et al..$^{33}$ & $1^{\circ} \mathrm{EF}$ ao $3^{\circ} \mathrm{EM}$ & $\uparrow$ & & $\uparrow$ & & & \\
\hline Schieffer et al. ${ }^{34}$ & EF e EM & $\uparrow$ & & $\uparrow$ & & & \\
\hline Schüller et al..$^{35}$ & 6 até 19 anos & & & & & $\uparrow$ & \\
\hline Slingerland et al..$^{36}$ & 7 até 15 anos & $\uparrow$ & & & & & \\
\hline Soriano-Férriz et al..$^{37}$ & 6 até 12 anos & & & $\uparrow$ & & & \\
\hline Tompsett et al. ${ }^{38}$ & 5 até 18 anos & $\uparrow$ & & $\uparrow$ & $\uparrow$ & & \\
\hline Trudeau et al..$^{39}$ & EF e EM & & & & $\uparrow$ & & \\
\hline Van de Kop et al..$^{40}$ & 12 até 17 anos & $\uparrow$ & & & & & \\
\hline Van Sluijs et al. ${ }^{41}$ & $1^{\circ} \mathrm{EF}$ ao $3^{\circ} \mathrm{EM}$ & $\uparrow$ & & & & & \\
\hline Vazou et al. ${ }^{42}$ & 5 até 18 anos & & & & & & $\uparrow$ \\
\hline Vella et al. ${ }^{43}$ & $1^{\circ} \mathrm{EF}$ ao $3^{\circ} \mathrm{EM}$ & & & & & & $\uparrow$ \\
\hline White et al. ${ }^{44}$ & NR & & & & $?$ & & \\
\hline Zach et al. ${ }^{45}$ & 5 até 18 anos & & & & & & $\uparrow$ \\
\hline \multicolumn{8}{|l|}{ Documentos } \\
\hline $\mathrm{CDC}^{46}$ & 5 até 18 anos & & & & & & $\uparrow$ \\
\hline $\mathrm{CDC}^{47}$ & $1^{\circ} \mathrm{EF}$ ao $3^{\circ} \mathrm{EM}$ & $\uparrow$ & & $\uparrow$ & $\uparrow$ & $\uparrow$ & \\
\hline $\mathrm{IOM}^{48}$ & $1^{\circ} \mathrm{EF}$ ao $3^{\circ} \mathrm{EM}$ & $\uparrow$ & & & $\uparrow$ & & $\uparrow$ \\
\hline PAGAC $^{49}$ & $1^{\circ} \mathrm{EF}$ ao $3^{\circ} \mathrm{EM}$ & $\uparrow$ & & & & & \\
\hline
\end{tabular}

\#AF = atividade física; ¥EF = ensino fundamental; ¥¥EM = ensino médio; NR = não reportou; CDC = Centro de Controle e Prevenção de Doenças; IOM = Instituto de Medicina (Washington, DC); PAGAC = Comissão Consultiva de Diretrizes de Atividade Física; $\uparrow$ = direção positiva do efeito; ? = resultados inconclusivos. 
res da saúde psicológica em três estudos; da saúde socioambiental e cognitiva em quatro estudos (Tabela 2).

Para responder a terceira pergunta, "Quais as estratégias relacionadas às aulas de EF escolar têm sido recomendadas para promoção de um estilo de vida ativo e saudável em escolares no mundo?" Um total de 2.408 documentos foram alcançados na busca. Após o processo de seleção, 63 documentos publicados de 2000 a 2020, foram analisados (lista de referências no material suplementar 3). Desses, um total de $27(42,8 \%)$ referiam-se à guias práticos; 19 (30,1\%) à posicionamentos; $10(15,9 \%)$ à recomendações e sete $(11,1 \%)$ à relatórios. A maioria deles era voltado aos gestores e políticos (62 documentos; $98,4 \%$ ), seguidos por professores (47 documentos;
$74,6 \%$ ), pais/familiares (28 documentos; 44,4\%) e estudantes (20 documentos; 31,7\%). Um quantitativo de estratégias considerável para a EF escolar foi encontrado na literatura. Após reuniões de consenso, foram compilados os seguintes quantitativos em cada dimensão: (i) política e ambiente: composta por sete recomendações com foco na valorização da EF escolar enquanto disciplina curricular obrigatória, nas condições de trabalho, na necessidade de ofertar uma EF de qualidade, pelo menos três aulas por semana (em todos os níveis de ensino da educação básica), e na necessidade de proporcionar formação continuada aos professores; (ii) currículo: quatro recomendações relacionadas ao tipo e progressão de conteúdos e coerência com referências

Tabela 2 - Efeito das aulas de EF escolar de intervenções brasileiras sobre o estilo de vida ativo e saudável de escolares

\begin{tabular}{|c|c|c|c|c|c|c|c|c|}
\hline \multicolumn{3}{|c|}{ Identificação do estudo } & \multicolumn{6}{|c|}{ Indicador de saúde } \\
\hline $\begin{array}{l}\text { Referência } \\
\text { (autor e ano) }\end{array}$ & Local/ Estado & $\begin{array}{c}\text { Ano escolar } \\
\left(\mathrm{EF}^{¥} \text { ou } \mathrm{EM}^{¥ ¥}\right)\end{array}$ & $\mathrm{AF} \#$ & $\begin{array}{c}\text { Outros } \\
\text { comportamentos* }\end{array}$ & $\begin{array}{l}\text { Saúde física/ } \\
\text { motota }\end{array}$ & $\begin{array}{c}\text { Saúde } \\
\text { psicológica }\end{array}$ & $\begin{array}{c}\text { Saúde } \\
\text { socioambiental }\end{array}$ & $\begin{array}{l}\text { Saúde cognitiva/ } \\
\text { desempenho escolar }\end{array}$ \\
\hline \multicolumn{9}{|c|}{ Intervenções focadas primariamente na educação física escolar } \\
\hline Cardeal et al. ${ }^{1}$ & $\begin{array}{l}\text { Taguatinga/ } \\
\text { DistritoFederal }\end{array}$ & $3^{\circ}$ e $4^{\circ}$ & & & $\uparrow$ & & & $\uparrow$ \\
\hline Coledam et al. $^{2}$ & $\begin{array}{l}\text { Nova } \\
\text { Europa/São Paulo }\end{array}$ & $5^{\circ} \mathrm{EF}$ & & & $\uparrow$ & & & \\
\hline Costa et al. ${ }^{3}$ & $\begin{array}{l}\text { Região do Cariri/ } \\
\text { Ceará }\end{array}$ & $1^{\circ}$ ao $4^{\circ} \mathrm{EF}$ & & & $\uparrow$ & & & \\
\hline Cunha et al. ${ }^{4}$ & São Paulo/ São Paulo & $1^{\circ}$ e $2^{\circ} \mathrm{EF}$ & $\uparrow$ & & & & & \\
\hline Farias et al. ${ }^{5,6}$ & $\begin{array}{l}\text { Porto Velho/ } \\
\text { Rondônia }\end{array}$ & $6^{\circ}$ ao $9^{\circ} \mathrm{EF}$ & & & $\uparrow$ & & & \\
\hline Finco et al. ${ }^{7}$ & $\begin{array}{l}\text { Porto Alegre/Rio } \\
\text { Grande do Sul }\end{array}$ & $\mathrm{EF}$ & $\uparrow$ & & & $\uparrow$ & $\uparrow$ & \\
\hline Gonzales et al. ${ }^{8}$ & $\begin{array}{l}\text { Canguçu/Rio } \\
\text { Grande do Sul }\end{array}$ & $5^{\circ} \mathrm{EF}$ ao $3^{\circ} \mathrm{EM}$ & & & & & & $\uparrow$ \\
\hline $\begin{array}{l}\text { Rossi Filho \& } \\
\text { Silva }^{9}\end{array}$ & Capivari/São Paulo & & & & & & $\uparrow$ & $\uparrow$ \\
\hline Silva et al. ${ }^{10}$ & Matinhos/Paraná & $3^{\circ}$ ao $5^{\circ} \mathrm{EF}$ & & & $\uparrow$ & & & \\
\hline Silveira et al. ${ }^{11}$ & São Paulo/São Paulo & $\mathrm{EF}$ (anos iniciais) & & & $\uparrow$ & & & \\
\hline Spohr et al. ${ }^{12}$ & Pelotas/RS & $5^{\circ} \mathrm{EF}$ ao $3^{\circ} \mathrm{EM}$ & & & & & & $\uparrow$ \\
\hline Oliveira et al. ${ }^{13}$ & Porto Alegre/RS & $1^{\circ} \mathrm{EM}$ & & & $\uparrow$ & & & \\
\hline Zanetti et al. ${ }^{14}$ & $\begin{array}{l}\text { São José do Rio } \\
\text { Pardo/São Paulo }\end{array}$ & NR & & & $\downarrow$ & & & \\
\hline \multicolumn{9}{|c|}{ Intervenções que integram a educação física escolar com outras estratégias (multicomponentes) } \\
\hline $\begin{array}{l}\text { Barbosa Filho et } \\
\text { al. }{ }^{15-18,18-21}\end{array}$ & Fortaleza/Ceará & $7^{\circ}$ ao $9^{\circ} \mathrm{EF}$ & $\uparrow$ & $\uparrow$ & & $\uparrow$ & $\uparrow$ & \\
\hline Nahas et al ${ }^{.22-28}$ & $\begin{array}{l}\text { Florianópolis/Santa } \\
\text { Catarina } \\
\text { Recife/Permanbuco }\end{array}$ & $1^{\circ}$ e $2^{\circ} \mathrm{EM}$ & $\uparrow$ & $\uparrow$ & $\uparrow$ & & & \\
\hline Knebel et al. ${ }^{29}$ & $\begin{array}{l}\text { Florianópolis/Santa } \\
\text { Catarina }\end{array}$ & $7^{\circ}$ ao $9^{\circ} \mathrm{EF}$ & & $\uparrow$ & & & & \\
\hline $\begin{array}{l}\text { Medeiros et } \\
\text { al. }{ }^{30-32}\end{array}$ & São Paulo/São Paulo & $1^{\circ}$ ao $3^{\circ} \mathrm{EM}$ & $\uparrow$ & $\uparrow$ & $\uparrow$ & $\uparrow$ & $\uparrow$ & \\
\hline $\begin{array}{l}\text { Minatto et } \\
\text { al. }{ }^{33-35}\end{array}$ & $\begin{array}{l}\text { Florianópolis/Santa } \\
\text { Catarina }\end{array}$ & $6^{\circ}$ aо $9^{\circ} \mathrm{EF}$ & & & & & & \\
\hline
\end{tabular}

\#AF = atividade física; ${ }^{\sharp} \mathrm{EF}=$ ensino fundamental; ${ }^{¥} \mathrm{EM}=$ ensino médio; NA = não se aplica; Formação = formação dos professores para atuação na EFE sobre AF e saúde; Outros* = outros comportamentos. 
nacionais, estaduais e municipais; (iii) instrução apropriada: obteve oito recomendações voltadas a inclusão dos alunos, utilização de recursos nas aulas e promoção da prática de atividade física; (iv) avaliação: composta por duas recomendações voltadas ao desenvolvimento e progressão de diferentes habilidades; (v) estratégias que interagem com a EF escolar: obteve cinco recomendações com informações adicionais aos alunos sobre as aulas de EF (Tabela 3). Maiores informações serão detalhadas em publicações futuras (in press).

A descrição do conteúdo gerado por meio da escuta foi fundamentada em duas matrizes: I - Contribuições da EF escolar para a saúde: a) foco em atividade física; b) em outros comportamentos à saúde; c) saúde física/ fisiológica; d) saúde psicológica; e) saúde social e ambiental; II - Recomendações por dimensão de análise:

Tabela 3 - Estratégias recomendadas para as aulas de educação física escolar na promoção do estilo de vida ativo e saudável.

\begin{tabular}{l} 
Recomendação \\
\hline A população brasileira deve reconhecer que a EF escolar é parte \\
importante das políticas de educação e saúde, e que ela contribui \\
para a saúde, o bem-estar e o desenvolvimento humano e social \\
das pessoas e do país ${ }^{1-6}$. \\
A EF escolar de qualidade deve ser obrigatória ao longo de todos \\
os anos da Educação Básica, incluindo a educação infantil \\
A comunidade escolar deve proporcionar e apoiar situações na \\
educação física com o mesmo rigor das outras disciplinas, por \\
exemplo, na aplicação de conteúdos, avaliação e desenvolvimento \\
profissional
\end{tabular}

A comunidade escolar deve garantir e valorizar condições de trabalho (materiais e espaços) e de qualificação profissional (formação especializada, continuada e apoio pedagógico) que favoreçam a qualidade das aulas de EF escolar $4,8,12,18,20,26,27,34-36,40$.

Considerem as escolas que valorizam o papel da educação física na formação de seu(sua) filho(a), ao longo de todos os anos de estudo $^{1-4,8,32}$

Deve-se assegurar pelo menos três aulas de educação física por semana, em todos os níveis da Educação Básica ${ }^{4,8-32}$.

Estabelecer frequência e duração mínima de EF: pelo menos $2 \mathrm{x} /$ semana ou $120 \mathrm{~min} / \mathrm{sem}$ para ensino fundamental e180 $\mathrm{min} / \mathrm{sem}$ para ensino médio; e, no mínimo, 30 minutos/sessão $7,10,11,14,16,22,24-27,29,30,35,41-45$
Os currículos devem apresentar uma estrutura sequencial e progressiva de objetivos de aprendizagem específicos da educação física, de acordo com referências nacionais, estaduais e municipais ${ }^{7,9,13,16,22,25,27,29,34,35}$.

As demais disciplinas da escola devem interagir com a educação física em conteúdos de educação em saúde $10,19,23,25,34,35,46,47$.

Envolvam-se ativamente nas aulas de EF escolar auxiliem o professor a escolher conteúdos e práticas que respeitem e desenvolvam a sua saúde física/motora, psicológica, social e cognitiva ${ }^{1,10,12,23,27,32,34,35,46,48}$.

Avançar para currículos baseados em competências que incorporem temas transversais aos conceitos-chave de educação física (ex., alfabetização digital, cidadania, meio ambiente, nutrição e sustentabilidade), apoiando a interdisciplinaridade.

\begin{tabular}{ll}
\hline & Instrução apropriada \\
\hline $\begin{array}{l}\text { Respeitem e acolham os colegas com diferentes habilidades, } \\
\text { deficiências e gostos durante as aulas, para contribuir na } \\
\text { construção de uma sociedade inclusiva e justa }\end{array}$ & $\begin{array}{l}\text { Respeite e acolha os colegas com diferentes habilidades, } \\
\text { deficiências e preferências durante as aulas. }\end{array}$ \\
\hline
\end{tabular}

Comunidade escolar

s currículos devem apresentar uma estrutura sequencia e progressiva de objetivos de aprendizagem específicos da

EF escolar, de acordo com referências nacionais, estaduais e municipais.

As demais disciplinas da escola também podem trabalhar com o movimento corporal, assim como a educação física pode explorar princípios matemáticos a partir de jogos.

Você deve se envolver ativamente nas aulas de EF escolar e auxiliar o professor a escolher conteúdos e práticas que respeitem e desenvolvam a sua saúde.

Alunos

Incorporem temas transversais relacionados à atividade física $\mathrm{e}$ saúde, como alfabetização digital, cidadania e sustentabilidade, para estimular a interdisciplinaridade.

Comunidade escolar

Professores

Professores
Política e ambiente

A comunidade escolar deve reconhecer que a educação física é parte importante das políticas de educação e saúde e que ela contribui para a saúde, a qualidade de vida e o desenvolvimento humano e social dos estudantes.

Para alcançar benefícios à saúde, a educação física de qualidade deve ser obrigatória ao longo de todos os anos da Educação Básica, incluindo a Educação Infantil.

A comunidade escolar deve proporcionar e apoiar a educação física da mesma forma que faz com as outras disciplinas, com a aplicação de conteúdos, avaliação e desenvolvimento profissional, por exemplo.

A comunidade escolar deve garantir e valorizar condições de trabalho (materiais e espaços) e de qualificação profissional (formação especializada, continuada e apoio pedagógico) que favoreçam a qualidade das aulas de EF escolar.

Para que essas orientações possam ser seguidas, são essenciais a qualificação e a valorização dos professores de educação física.

Escolham as escolas que valorizam o papel da educação física na formação da criança e do jovem ao longo de todos os anos de estudo.

Devem ser oferecidas pelo menos três aulas de educação física de 50 minutos cada, por semana.

Quais são as recomendações para as aulas de educação física?

Comunidade escolar

Quais são as recomendações para as aulas de educação física?

Comunidade escolar

Comunidade escolar

Quais são as recomendações para as aulas de educação física?

Pais ou responsáveis 
Continuação da Tabela 3 - Estratégias recomendadas para as aulas de educação física escolar na promoção do estilo de vida ativo e saudável.

\begin{tabular}{|c|c|c|}
\hline Recomendação & Tradução para a população brasileira & Seção do Guia \\
\hline $\begin{array}{l}\text { Incentivem seu(sua) filho(a) a explorar as atividades aprendidas } \\
\text { nas aulas de educação física, para além da escola, com foco em } \\
\text { uma vida ativa e saudável ao longo da vida }{ }^{4,9,10,18-21,25,27,32,34} \text {. }\end{array}$ & $\begin{array}{l}\text { Incentivem a criança e o jovem a explorar as atividades } \\
\text { aprendidas nas aulas de educação física, para além da escola, com } \\
\text { foco em uma vida ativa e saudável ao longo da vida. }\end{array}$ & Pais ou responsáveis \\
\hline $\begin{array}{l}\text { Busquem melhorar a qualidade das aulas, por meio de formações } \\
\text { e trocas de experiências sobre o currículo, práticas pedagógicas e } \\
\text { avaliação }{ }^{25,32,34,36} \text {. }\end{array}$ & $\begin{array}{l}\text { Busquem melhorar a qualidade das suas aulas por meio de } \\
\text { formações e trocas de experiências sobre o currículo, práticas } \\
\text { pedagógicas e avaliação. }\end{array}$ & Professores \\
\hline $\begin{array}{l}\text { Adotem estratégias que mantenham os estudantes ativos e } \\
\text { participativos na maior parte da aula, por meio de práticas } \\
\text { pedagógicas diversificadas, interativas, desafiadoras, prazerosas e } \\
\text { que estimulem a confiança e autonomia dos estudantes } 9,10,13,15,16,1 \\
9,22,25,26,29,30,34,39,41,45,49\end{array}$ & $\begin{array}{l}\text { Adotem estratégias que mantenham os estudantes fisicamente } \\
\text { ativos e participativos na maior parte da aula, por meio de práticas } \\
\text { pedagógicas diversificadas, interativas, desafiadoras, que tragam } \\
\text { satisfação e que estimulem a confiança e autonomia dos estudantes. }\end{array}$ & Professores \\
\hline $\begin{array}{l}\text { Abordem a temática da atividade física e suas relações com } \\
\text { os saberes da cultura e expressão corporal, promovendo } \\
\text { discussões sobre desigualdades sociais, como etnias e gênero, e } \\
\text { determinantes de saúde }{ }^{25,31,34,35,47} \text {. }\end{array}$ & $\begin{array}{l}\text { Abordem a temática da atividade física e suas relações com } \\
\text { os saberes da cultura e expressão corporal, respeitando as } \\
\text { especificidades locais e regionais, para promover discussões } \\
\text { também sobre desigualdades sociais, como etnias e gênero, e } \\
\text { determinantes de saúde. }\end{array}$ & Professores \\
\hline $\begin{array}{l}\text { Proporcionem o envolvimento de todos os estudantes nas aulas, } \\
\text { independentemente de habilidades, deficiências e condições de } \\
\text { saúde } \text { f }^{4,10,16,22,23,25,27,29,32,34,35,39,47} \text {. }\end{array}$ & $\begin{array}{l}\text { Proporcionem o envolvimento de todos os estudantes nas aulas, } \\
\text { independentemente de habilidades, deficiências e condições de } \\
\text { saúde. }\end{array}$ & Professores \\
\hline $\begin{array}{l}\text { Utilizem tecnologias e estratégias pedagógicas inovadoras nas } \\
\text { aulas, bem como estimulem a participação dos estudantes em } \\
\text { aulas de campo e atividades na comunidade }{ }^{25,32,34,46} \text {. }\end{array}$ & $\begin{array}{l}\text { Utilizem tecnologias e estratégias pedagógicas inovadoras nas } \\
\text { aulas, bem como estimulem a participação dos estudantes em } \\
\text { aulas e atividades na comunidade. }\end{array}$ & Professores \\
\hline $\begin{array}{l}\text { O professor de Educação Física deve usar tecnologias e } \\
\text { abordagens inovadoras (ex. jogos eletrônicos, aplicativos, sistemas } \\
\text { e outros), tanto no desenvolvimento das aulas, como no processo } \\
\text { de avaliação dos estudantes } \text { s }^{25,32,34,46} \text {. }\end{array}$ & $\begin{array}{l}\text { As aulas devem incluir conteúdos que possibilitem experiências } \\
\text { positivas e abordagens inovadoras para os estudantes. }\end{array}$ & $\begin{array}{l}\text { Quais são as } \\
\text { recomendações para as } \\
\text { aulas de educação física? }\end{array}$ \\
\hline $\begin{array}{l}\text { Construam um ambiente favorável para que os alunos se sintam } \\
\text { motivados a vivenciar as aulas de educação física }{ }^{32,46,50} \text {. }\end{array}$ & $\begin{array}{l}\text { Construam um ambiente favorável para que os alunos se sintam } \\
\text { motivados a vivenciar as aulas de educação física }{ }^{32,46,50} \text {. }\end{array}$ & Professores \\
\hline $\begin{array}{l}\text { Construam um ambiente favorável para que os alunos se sintam } \\
\text { motivados a vivenciar as aulas de educação física }{ }^{32,46,50} \text {. }\end{array}$ & $\begin{array}{l}\text { Construam um ambiente favorável para que os alunos se sintam } \\
\text { motivados a vivenciar as aulas de educação física }{ }^{32,46,50} \text {. }\end{array}$ & Alunos \\
\hline
\end{tabular}

Participem das aulas de EF escolar para desenvolver conhecimentos, habilidades e competências para fazer diferentes tipos de atividade física (esportes, jogos, brincadeiras, ginásticas, danças e lutas) $)^{9,10}$.

Estimulem a participação ativa e acompanhem o progresso de seu(sua) filho(a) nas aulas de EF escolar para que ele desenvolva conhecimentos, habilidades e competências para fazer diferentes tipos de atividade física $22,25,29,32,34,35,41$.
Participe das aulas de EF escolar para desenvolver habilidades e para conhecer diferentes tipos de atividade física. Isso vai ajudar você a ter uma vida ativa!

Estimulem a participação ativa e acompanhem o progresso da criança e do jovem nas aulas de educação física;
Alunos

Pais ou responsáveis

Estratégias que interagem com a EFE

Fique atento (a) às orientações do professor durante as aulas e aos ambientes de prática para sua segurança e a de seus colegas $34,35,42,43,51,52$.

Não tenha vergonha de participar das aulas de EF escolar, o professor e seus colegas vão ajudar você no aprendizado das atividades.

Se for possível, use roupas leves e calçados adequados e confortáveis para a prática de atividade física.

Você deve beber água antes, durante e após a prática de atividade física. Também deve consumir uma alimentação saudável e para saber mais sobre esse tema, consulte o Guia Alimentar para a População Brasileira ${ }^{16,24,42,43,47}$.

Ao praticar atividade física ao ar livre, utilize alguma forma de proteção, como boné, camisa de manga longa e protetor solar, se possível.
Fique atento (a) às orientações do professor durante as aulas e aos ambientes de prática para sua segurança e a de seus colegas.

Não tenha vergonha de participar das aulas de EF escolar, o professor e seus colegas vão ajudar você no aprendizado das atividades.

Se for possível, use roupas leves e calçados adequados e confortáveis para a prática de atividade física.

Você deve beber água antes, durante e após a prática de atividade física. Também deve consumir uma alimentação saudável e para saber mais sobre esse tema, consulte o Guia Alimentar para a População Brasileira.

Ao praticar atividade física ao ar livre, utilize alguma forma de proteção, como boné, camisa de manga longa e protetor solar, se possível.
Alunos

Alunos

Orientações adicionais aos alunos

Orientações adicionais aos alunos

Orientações adicionais aos alunos

$\mathrm{EF}=$ educação física.

a) política e meio ambiente; b) currículo; c) instrução apropriada; d) avaliação; e) ações escolares relacionadas à educação física. Tais informações foram obtidas de esco- lares $(n=24)$, gestores $(n=20)$, professores de educação física $(n=20)$, e pais/responsáveis $(n=11)$ - Tabela 4 .

$\mathrm{O}$ compilado das evidências encontradas nas etapas 
descritas acima possibilitaram a criação de recomendações para as aulas de EF escolar para a população brasileira. A mensagem principal é:

Devem ser oferecidas, por semana, pelo menos três aulas de educação física escolar de $\mathbf{5 0}$ minutos cada uma. As aulas devem incluir conteúdos que possibilitem experiências positivas e abordagens inovadoras para os estudantes.

Orientações para os estudantes e recomendações para professores, pais e responsáveis e a comunidade escolar de uma forma geral podem ser visualizadas na Tabela 3.

\section{Discussão}

As etapas de elaboração do capítulo de EF escolar apresentado no Guia foram descritas no presente artigo. As sínteses de evidências científicas indicaram que as aulas de EF escolar contribuíram para diferentes dimen- sões da saúde, com ênfase para a saúde física/motora. Inúmeras estratégias têm sido testadas, e a mensagem principal apontou para a necessidade de garantir pelo menos três aulas por semana de EF escolar de qualidade em todos os anos da Educação Básica.

Resultados da primeira revisão chamam atenção para os inúmeros benefícios à saúde advindos da participação em aulas de EF escolar ${ }^{3-11}$. Ao ouvir a comunidade escolar, foi unânime o reconhecimento de que a EF escolar contribui para a saúde. Os estudantes relataram que a $\mathrm{EF}$ escolar contribui para incentivar a prática de atividade física e traz benefícios à saúde física/motora, psicológica e socioambiental. Na percepção dos gestores, a prática de atividade física melhora a qualidade de vida, o bem-estar social, a disposição diária, a saúde mental e estimula hábitos saudáveis. Pais e responsáveis relataram que a EF escolar incentiva a permanência na atividade física ao longo da vida;

Tabela 4 - Percepção da contribuição e da importância das aulas de educação física (EF) escolar para a saúde e recomendações sugeridas por dimensões abordadas.

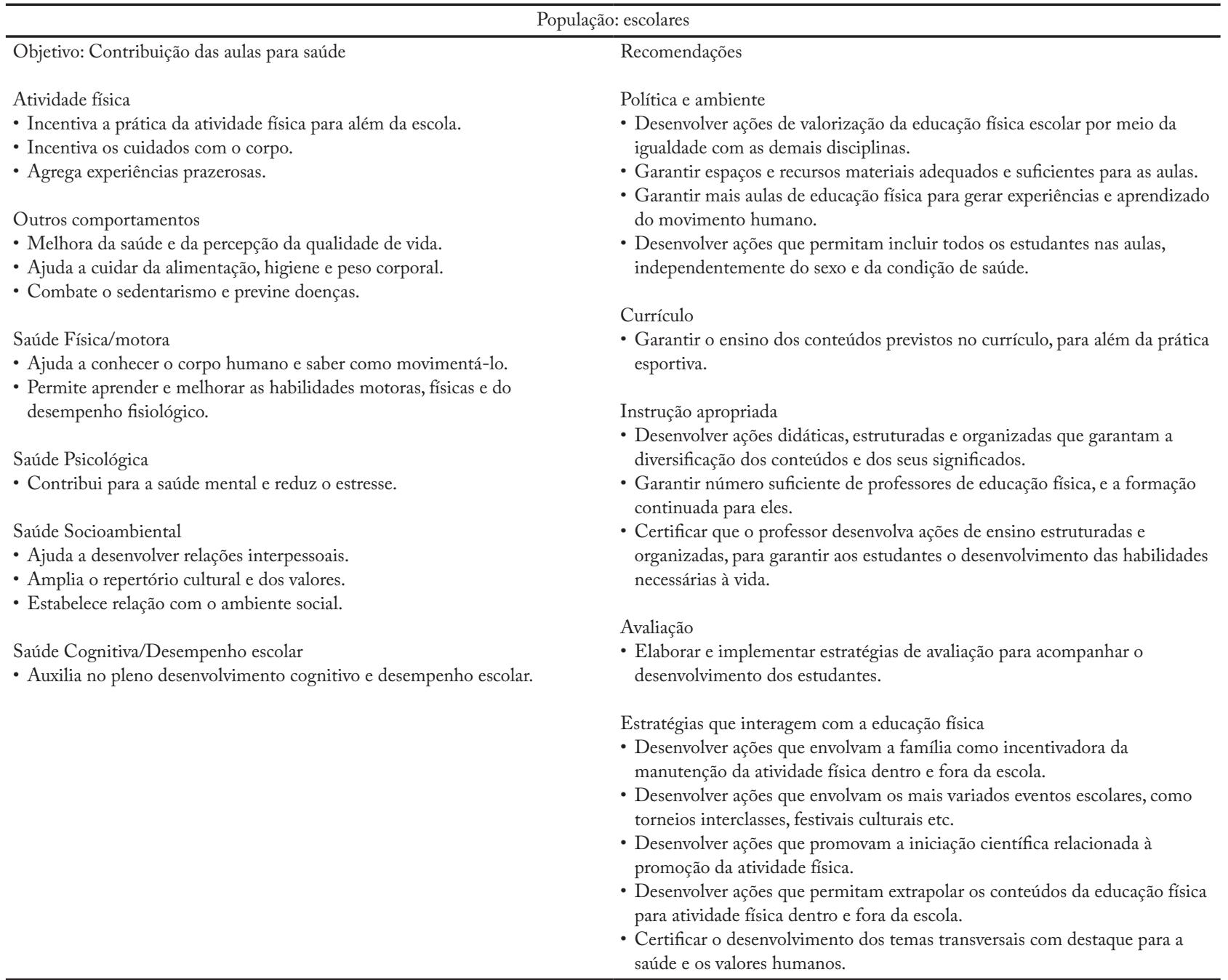


Continuação da Tabela 4 - Percepção da contribuição e da importância das aulas de educação física (EF) escolar para a saúde e recomendações sugeridas por dimensões abordadas.

\begin{tabular}{|c|c|}
\hline \multicolumn{2}{|c|}{ População: gestores } \\
\hline Objetivo: Contribuição das aulas para saúde & Recomendações \\
\hline $\begin{array}{l}\text { Atividade física } \\
\text { - Incentiva o não sedentarismo. } \\
\text { - Incentiva a atividade física. } \\
\text { Outros comportamentos } \\
\text { - Melhora a qualidade de vida. } \\
\text { - Incentiva hábitos saudáveis. } \\
\text { - Previne diversas doenças. }\end{array}$ & $\begin{array}{l}\text { Política e ambiente } \\
\text { - Aumento e melhoria dos espaços físicos, equipamentos e materiais } \\
\text { disponíveis. } \\
\text { - Capacitação dos professores, elaboração de aulas dinâmicas e atrativas; } \\
\text { que enfatize a importância da atividade física; da alimentação saudável; e } \\
\text { trabalhe conteúdos científicos produzidos a nível escolar. } \\
\text { - Distribuição e aumento do número de professores. } \\
\text { - Adesão e apoio aos projetos sugeridos pelos profissionais. } \\
\text { - Aumento do número de aulas de educação física na semana. }\end{array}$ \\
\hline $\begin{array}{l}\text { Saúde Física/motora } \\
\text { - Melhora a saúde, o condicionamento, o desenvolvimento de habilidades } \\
\text { físicas. } \\
\text { - Proporciona conhecimento sobre as habilidades corporais. }\end{array}$ & $\begin{array}{l}\text { - Criação de diretrizes para as aulas de educação física. } \\
\text { Currículo } \\
\text {--- }\end{array}$ \\
\hline $\begin{array}{l}\text { Saúde Psicológica } \\
\text { - Melhora a disposição diária. } \\
\text { - Melhora a saúde mental. }\end{array}$ & $\begin{array}{l}\text { Instrução apropriada } \\
\text {--- } \\
\text { Avaliacão }\end{array}$ \\
\hline $\begin{array}{l}\text { Saúde Socioambiental } \\
\text { - Melhora o bem-estar social. } \\
\text { - Proporciona socialização. }\end{array}$ & Estratégias que interagem com a educação física \\
\hline $\begin{array}{l}\text { Saúde Cognitiva/Desempenho escolar } \\
\text { - Melhora as habilidades motoras. } \\
\text { - Melhora o desenvolvimento cognitivo. }\end{array}$ & $\begin{array}{l}\text { - Implementação do Programa Saúde do Escolar. } \\
\text { - Promoção de jogos escolares; feiras educacionais e eventos de intercâmbios. }\end{array}$ \\
\hline População: pais e responsáveis & População: professores de educação física \\
\hline Objetivo: Contribuições das aulas para saúde & Objetivo: Fatores que dificultam a prática pedagógica \\
\hline $\begin{array}{l}\text { Atividade física } \\
\text { - Vida adulta ativa. } \\
\text { - Adiciona a atividade física em alternância as aulas teóricas de sala. }\end{array}$ & $\begin{array}{l}\text { Política e ambiente } \\
\text { - Aumento do investimento para capacitações dos professores. } \\
\text { - Aumento de investimento para melhoria da estrutura escolar. }\end{array}$ \\
\hline $\begin{array}{l}\text { Outros comportamentos } \\
\text { - Combate ao sedentarismo. } \\
\text { - Estimula hábitos saudáveis. } \\
\text { - Para ter boa saúde. }\end{array}$ & $\begin{array}{l}\text { - Ampliar a interação com as universidades. } \\
\text { - Reduzir o número de alunos por turma. } \\
\text { - Melhorar a infraestrutura e materiais. }\end{array}$ \\
\hline - Prevenção de doenças. & $\begin{array}{l}\text { Currículo } \\
\text { - Padronizar os conteúdos. }\end{array}$ \\
\hline $\begin{array}{l}\text { Saúde Física/motora } \\
\text { - Desenvolve a coordenação motora. } \\
\text { - Ajuda no crescimento e desenvolvimento. }\end{array}$ & $\begin{array}{l}\text { - Incentivar a Interdisciplinaridade. } \\
\text { - Aprofundar o conhecimento sobre o currículo. }\end{array}$ \\
\hline Saúde Psicológica & $\begin{array}{l}\text { Instrução apropriada } \\
\text { - Melhorar as capacitações sobre métodos de ensino. } \\
\text { - Realizar mais cursos de formação e reciclagem para novos aprendizados } \\
\text { - Diversificar as metodologias. }\end{array}$ \\
\hline $\begin{array}{l}\text { Saúde Socioambiental } \\
\text { • Interação e socialização com os colegas. }\end{array}$ & - Aplicar os conteúdos do currículo. \\
\hline - Valores e normas. & Avaliação \\
\hline $\begin{array}{l}\text { Saúde Cognitiva/Desempenho escolar } \\
---\end{array}$ & $\begin{array}{l}\text { Estratégias que interagem com a educação física } \\
\text {--- }\end{array}$ \\
\hline
\end{tabular}

contribui para adoção de comportamentos saudáveis e para a saúde social. Isto foi importante para demonstrar que as evidências científicas foram validadas pelos membros da comunidade escolar, reconhecendo os benefícios da participação em aulas de EF escolar para a saúde do estudante.
As estratégias para EF escolar foram analisadas seguindo a proposta do America-Society of Health and Physical Educators ${ }^{12}$. Em 63 documentos publicados, o presente estudo verificou que as estratégias mais utilizadas são aquelas que interagem com a EF escolar, seguida por política e ambiente, instrução apropriada, currículo e 
avaliação. O público-alvo, em sua maioria, são gestores e políticos, seguido por professores, pais/familiares e estudantes. No âmbito da política e ambiente foram apontadas estratégias como a necessidade de: (a) valorização da EF escolar (estudantes), (b) estrutura e materiais (estudantes, gestores e professores), (c) mais aulas de EF escolar (estudantes, gestores), (d) presença e postura do professor (estudantes), (e) capacitação dos professores (gestores e professores), (f) distribuição e aumento do número de professores (gestores), e (g) redução do número de alunos em sala de aula (professores).

Com relação ao currículo, foram mencionados a necessidade de: (a) garantir o ensino dos conteúdos previstos no currículo, para além da prática esportiva (estudantes), (b) padronizar o conteúdo e viabilizar a interdisciplinaridade (professores). Para instrução apropriada, atenção especial deve ser dada a diversificação de conteúdos (estudantes) e a capacitação dos professores (estudantes e professores). Quanto à avaliação, considera-se a implementação de estratégias que possibilitem acompanhar o desenvolvimento do aluno (estudantes). Quando questionadas as estratégias que interagem com a EF escolar, foram relatadas a importância de: (a) desenvolver ações que envolvam a família; (b) trabalhar temas voltados à saúde e valores humanos (estudantes); (c) desenvolver eventos e jogos escolares (estudantes e gestores). Em síntese, houve ressonância entre as principais estratégias mencionadas pela literatura e relatadas na escuta, com foco na dimensão política e ambiente para garantir uma EF escolar suficiente e de qualidade.

Diferentemente de algumas recomendações de atividade física publicadas em outros países, o Guia traz como inovação a inclusão da EF escolar. Esta iniciativa é importante para nortear a agenda das políticas públicas do Brasil voltadas para promoção da atividade física e de outros indicadores de saúde na população. A mensagem principal para gestores e políticos é que investir na EF escolar pode ser o melhor caminho para promover benefícios imediatos na saúde da criança e do adolescente, e contribuir para uma mudança de paradigma e de cultura das gerações futuras. Isso acontece porque, na maioria das vezes, a primeira oportunidade teórico-prática de atividade física estruturada que a criança acessa é a EF escolar. Conhecer e vivenciar a cultura do movimento corporal e seus benefícios à saúde já na educação infantil, repercutirá em uma sociedade não apenas fisicamente ativa, mas também saudável.

No campo da pesquisa, recomenda-se a ampliação de estudos que testem associações da EF escolar com a saú- de psicológica e cognitiva/desempenho escolar, dada a menor quantidade de estudos encontrados. Com relação às estratégias, é chegada a hora de testar a implementação e avaliar a efetividade das estratégias aqui apresentadas em diferentes cenários e desfechos em saúde. Além disso, é fundamental que o Guia seja disseminado para além da academia, pois recomendações específicas foram sumarizadas para toda a comunidade escolar.

A compilação de evidências acerca do efeito da participação em aulas de EF escolar sobre a saúde de crianças e adolescentes e as principais estratégias utilizadas para alcançar esses benefícios constitui parte do Guia de Atividade Física para a População Brasileira. Em síntese, recomenda-se garantir o acesso à EF escolar em todos os ciclos da Educação Básica Brasileira, no mínimo três aulas por semana, obrigatórias e de qualidade, ministradas por professores com formação em Educação Física. Tal meta somente será alcançada se todos os setores que compõem o sistema trabalharem conjuntamente, com atenção especial para políticos e gestores, como principais atores das tomadas de decisão para elaboração de políticas, planos de ação e programas para mudança da realidade.

\section{Conflito de interesse}

Os autores declaram não haver conflito de interesse.

\section{Financiamento}

Este trabalho integra o Guia de Atividade Física para a População Brasileira, que foi financiado pelo Ministério da Saúde do Brasil por meio do Termo de Execução Descentralizada n ${ }^{\circ}$ 56/2019 (projeto: 79224219002/2019; processo: 25000.171034/2019-27).

\section{Contribuição dos autores}

Silva KS, participou da concepção e redação do manuscrito, análise e interpretação dos dados. Barbosa Filho VC, Camargo EM, Oliveira VJM, Ravagnani FCP, Sandreschi PF, Santos PC, Silva $\mathrm{AB}$, Ramires VV, Tenório MCM, participaram da concepção, coleta, análise de dados, revisão crítica e aprovação do manuscrito. Hallal PC participou da concepção, redação e revisão geral do manuscrito. Todos os autores aprovaram a versão final do manuscrito.

\section{Referências}

1. Brasil. LEI No 9.394, DE 20 DE DEZEMBRO DE 1996 - Lei de diretrizes e bases da educação nacional [Internet]. 1996 [citado 2020 out 9]. Disponível em: http://www. planalto.gov.br/ccivil_03/Leis/L9394.htm.

2. Brasil. Base Nacional Comum Curricular [Internet]. Ministério da Educação; 2018 [citado 2020 out 9]. Disponível em: http://basenacionalcomum.mec.gov.br/images/BNCC_ EI_EF_110518_versaofinal_site.pdf. 
3. Tompsett C, Sanders R, Taylor C, Cobley S. Pedagogical approaches to and effects of fundamental movement skill interventions on health outcomes: a systematic review. Sports Med. 2017;47(9):1795-819.

4. Educating the Student Body: Taking Physical Activity and Physical Education to School. Washington, D.C.: National Academies Press; 2013 [citado 2020 jul 14]. Disponível em: http://www.nap.edu/catalog/18314.

5. Centers for Disease Control and Prevention (CDC). School Health Guidelines to Promote Healthy Eating and Physical Activity. 60(5).

6. Dudley D, Okely A, Pearson P, Cotton W. A systematic review of the effectiveness of physical education and school sport interventions targeting physical activity, movement skills and enjoyment of physical activity. Eur. Phys. Educ. Rev. 2011;17(3):353-78.

7. Bessa C, Hastie P, Araújo R, Mesquita I. What do we know about the development of personal and social skills within the sport education model: a systematic review. J Sports Sci Med. 2019;18(4):812-29.

8. Gomes GMG, Gomes FRH, Ribeiro PS. Self-concern, physical education and students from 6 to 10 years: a systematic review of the literature. Cad. Educ. Fis. Esp. 2019;17(2):1-10.

9. Braithwaite R, Spray CM, Warburton VE. Motivational climate interventions in physical education: A meta-analysis. Psychol Sport Exerc. 2011;12(6):628-38.

10. Trudeau F, Shephard RJ. Physical education, school physical activity, school sports and academic performance. Int J Behav Nutr Phys Act. 2008;5(1):10.

11. White RL, Babic MJ, Parker PD, Lubans DR, Astell-Burt T, Lonsdale C. Domain-specific physical activity and mental health: a meta-analysis. Am. J. Prev. Med.2017;52(5):653-66.

12. SHAPE America-Society of Health and Physical Educators. The essential components of physical education. Author Reston, VA; 2015.

13. UNESCO. Diretrizes em educação física de qualidade (EFQ) para gestores de políticas - UNNESCO Digital Library. 2015 [citado 2020 maio 18]. Disponível em: https://unesdoc. unesco.org/ark:/48223/pf0000231963

14. Centers for Disease Control and Prevention. A CDC Review of School Laws and Policies Concerning Child and Adolescent Health. J. Sch. Health. 2008;78(2):69-128.

15. Spence JC, Faulkner G, Bradstreet CC, Duggan M, Tremblay MS. Active Canada 20/20: A physical activity plan for Canada. Can J Public Health.2015;106:470-73.

16. The Australian Council for Health, Physical Education and Recreation (ACHPER): Support of the Australian Curriculum: Health and Physical Education. 2017 [citado 2020 jul 13]. Disponível em: https://www.achper.org.au/ documents/item/393.

17. The common Wealth. Model indicators on sport, physical education and physical activity and the Sustainable Development Goals. 2019 [citado 2020 jul 5]. Disponível em: https://thecommonwealth.org/sites/default/files/inline/ Sport\%20and\%20SDG\%20Indicators\%20v3.1.pdf.

18. Centers for Disease Control and Prevention (CDC). School health guidelines to promote healthy eating and physical activity. MMWR Recomm Rep. 2011;60(RR-5):1-76.
19. Opstoel K, Chapelle L, Prins FJ, De Meester A, Haerens $\mathrm{L}$, van Tartwijk J, et al. Personal and social development in physical education and sports: A review study. Eur. Phys. Educ. Rev. 2020;26(4):797-813.

20. Schüller I, Demetriou Y. Physical activity interventions promoting social competence at school: A systematic review. Educ. Res. Rev. 2018;25:39-55.

21. Rasberry CN, Lee SM, Robin L, Laris BA, Russell LA, Coyle KK, et al. The association between school-based physical activity, including physical education, and academic performance: a systematic review of the literature. Prev Med. 2011;52(Suppl 1):S10-20.

22. Vazou S, Pesce C, Lakes K, Smiley-Oyen A. More than one road leads to Rome: A narrative review and meta-analysis of physical activity intervention effects on cognition in youth. Int J Sport Exerc Psychol. 2019;17(2):153-78.

23. Barba-Martín RA, Bores-García D, Hortigüela-Alcalá D, González-Calvo G. The application of the teaching games for understanding in physical education. systematic review of the last six years. Int. J. Environ. Res. Public Health. 2020;17(9):3330.

24. Vella SA, Braithewaite RE, Gardner LA, Spray CM. A systematic review and meta-analysis of implicit theory research in sport, physical activity, and physical education. Int Rev Sport Exerc Psychol. 2016;9(1):191-214.

25. Brasil. Casa Civil, Subchefia para Assuntos Jurídicos. Decreto no 6.286, de 5 de dezembro de 2007. Institui o Programa Saúde na Escola - PSE, e dá outras providências. Diário Oficial da União; 2007.

26. Brasil. Ministério da Educação, Gabinete do Ministro. Portaria Interministerial no 3.497 , de 24 de novembro de 2003. Diário Oficial da União; 2003.

27. Brasil. Ministério da Saúde. Secretaria de Atenção Primária à Saúde. Departamento de Promoção da Saúde. Guia de Atividade Física para a População Brasileira / Ministério da Saúde, Secretaria de Atenção Primária à Saúde, Departamento de Promoção da Saúde. - Brasília : Ministério da Saúde, 2021.

28. Tricco AC, Lillie E, Zarin W, O’Brien KK, Colquhoun H, Levac D, et al. PRISMA Extension for Scoping Reviews (PRISMA-ScR): Checklist and Explanation. Ann Intern Med. 2018;169(7):467.

29. Barbosa Filho VC, da Silva KS, Mota J, Beck C, Lopes A da S. A physical activity intervention for Brazilian students from low human development index areas: A cluster-randomized controlled trial. J Phys Act Health. 2016;13(11):1174-82.

30. Biddle SJ, O'Connell S, Braithwaite RE. Sedentary behaviour interventions in young people: a meta-analysis. Br. J. Sports Med. 2011;45(11):937-42.

Recebido: $28 / 01 / 2021$

Aprovado: 10/04/2021

Como citar este artigo:

Silva KS, Bandeira AS, Ravagnani FCP, Camargo EM, Tenório MC, Oliveira VJM, Santos PC, Ramires VV, Sandreschi PF, Hallal PC, Barbosa Filho VC. Educação física escolar: Guia de Atividade Física para a População Brasileira. Rev Bras Ativ Fís Saúde. 2021;26:e0219. DOI. 10.12820/rbafs.26e0219 


\section{Material suplementar 1}

\section{Lista de referências incluídas para a questão 1 " $O$ que} as sinteses de evidências relatam sobre as contribuiçôes das aulas de EF escolar para diferentes componentes do estilo de vida ativo e saudável em estudantes?"

1. Álvarez-Bueno C, Pesce C, Cavero-Redondo I, SánchezLópez M, Garrido-Miguel M, Martínez-Vizcaíno V. Academic Achievement and Physical Activity: A Metaanalysis. Pediatrics. 2017;140(6):e20171498.

2. Barba-Martín RA, Bores-García D, Hortigüela-Alcalá D, González-Calvo G. The application of the teaching games for understanding in physical education. systematic review of the last six years. IJERPH. 2020;17(9):3330.

3. Becerra-Fernández, Carlos Alberto, Mayorga-Vega, Daniel, Merino-Marban, Rafael. Effect of Physical Education-based stretching programs on hamstring extensibility in high school students: A systematic review. CCD. 2020;15(43):63-73.

4. Becerra Fernandez C-A, Merino-Marban R. Efficacy of hamstring stretching programs in schoolchildren. A systematic review. Timisoara Physical Education and Rehabilitation Journal. 2015;8(15):36-43.

5. Bessa C, Hastie P, Araújo R, Mesquita I. What Do We Know About the development of personal and social skills within the sport education model: a systematic review. 2019;18.

6. Braithwaite R, Spray CM, Warburton VE. Motivational climate interventions in physical education: A meta-analysis. Psychol Sport Exerc. 2011;12(6):628-38.

7. Camacho-Minano MJ, LaVoi NM, Barr-Anderson DJ. Interventions to promote physical activity among young and adolescent girls: a systematic review. Health Educ. Res. 2011;26(6):1025-49.

8. Chacón-Borrego F, Ubago-Jimenez JL. Educación e higiene postural en el ámbito de la Educación Física. Papel del maestro en la prevención de lesiones. Revisión sistemática Education and postural hygiene in the field of physical education. Teacher's role in injury prevention. Systematic review. 2018;6.

9. Dobbins M, Husson H, DeCorby K, LaRocca RL. Schoolbased physical activity programs for promoting physical activity and fitness in children and adolescents aged 6 to 18. Cochrane Metabolic and Endocrine Disorders Group, organizador. Cochrane Database of Systematic Reviews [Internet].28 de fevereiro de 2013 [citado 13 de julho de 2020]; Disponível em: http://doi.wiley.com/10.1002/14651858. CD007651.pub2

10. Donnelly JE, Hillman CH, Castelli D, Etnier JL, Lee S, Tomporowski P, et al. Physical Activity, Fitness, Cognitive Function, and Academic Achievement in Children: A Systematic Review. Med Sci Sports Exerc. 2016;48(6):1197-222.

11. Dudley D, Burden R. What effect on learning does increasing the proportion of curriculum time allocated to physical education have? A systematic review and meta-analysis. Eur. Phys. Educ. Rev. 2020;26(1):85-100.

12. Dudley D, Okely A, Pearson P, Cotton W. A systematic review of the effectiveness of physical education and school sport interventions targeting physical activity, movement skills and enjoyment of physical activity. Eur. Phys. Educ. Rev. 2011;17(3):353-78.

13. Gomes GMG, Gomes FRH, Ribeiro PS. Self-concern, physical education and students from 6 to 10 years: a systematic review of the literature. P S. 2019;17(2):7.
14. Gunnell KE, Poitras VJ, LeBlanc A, Schibli K, Barbeau K, Hedayati N, et al. Physical activity and brain structure, brain function, and cognition in children and youth: A systematic review of randomized controlled trials. Ment. Health Phys Act. 2019;16:105-27.

15. García-Hermoso A, Alonso-Martínez AM, Ramírez-Vélez R, Pérez-Sousa MÁ, Ramírez-Campillo R, Izquierdo M. Association of physical education with improvement of health-related physical fitness outcomes and fundamental motor skills among youths: a systematic review and metaanalysis. JAMA Pediatr. 2020;174(6):e200223.

16. Hoehner CM, Ribeiro IC, Parra DC, Reis RS, Azevedo MR, Hino AA, et al. Physical activity interventions in Latin America: expanding and classifying the evidence. Am J Prev Med. 2013;44(3):e31-40.

17. Jenkinson KateA, Naughton G, Benson AC. Peer-assisted learning in school physical education, sport and physical activity programmes: a systematic review. Physical Education and Sport Pedagogy. 2014;19(3):253-77.

18. Kahn EB, Ramsey LT, Brownson RC, Heath GW, Howze $\mathrm{EH}$, Powell KE, et al. The effectiveness of interventions to increase physical activity. Am J Prev Med. 2002;35.

19. Kopp D, Prat I, Azevedo M. Intervenções escolares de médio e longo prazo para promoção de atividade física: Revisão sistemática. Rev Bras Ativ Fis Saude [Internet]. 31 de março de 2014 [citado 13 de julho de 2020];19(2). Disponível em: http://periodicos.ufpel.edu.br/ojs2/index.php/RBAFS/ article/view/3896

20. Lai SK, Costigan SA, Morgan PJ, Lubans DR, Stodden DF, Salmon J, et al. Do school-based interventions focusing on physical activity, fitness, or fundamental movement skill competency produce a sustained impact in these outcomes in children and adolescents? A systematic review of follow-up studies. Sports Med. 2014;44(1):67-79.

21. Lambrinou, Christina-Paulina, Androutsos O, Karaglani E, Cardon G, Huys N, Wikström K, et al. Effective strategies for childhood obesity prevention via school based, family involved interventions: a critical review for the development of the Feel4Diabetes-study school based component. BMC Endocr Disord. 2020;20(S2):52.

22. Lonsdale C, Rosenkranz RR, Peralta LR, Bennie A, Fahey P, Lubans DR. A systematic review and meta-analysis of interventions designed to increase moderate-to-vigorous physical activity in school physical education lessons. Preventive Medicine. 2013;56(2):152-61.

23. McDonald SM, Clennin MN, Pate RR. Specific strategies for promotion of physical activity in kids-which ones work? a systematic review of the literature. Am. J. Lifestyle Med. 2018;12(1):51-82.

24. Marques A, Gómez F, Martins J, Catunda R, Sarmento $\mathrm{H}$, de Lisboa U. Association between physical education, school-based physical activity, and academic performance: a systematic review Asociación entre la educación física, la actividad física en la escuela, y el rendimiento académico: una revisión sistemática. 2017;6.

25. Matson-Koffman DM, Brownstein JN, Neiner JA, Greaney ML. A Site-specific literature review of policy and environmental interventions that promote physical activity and nutrition for cardiovascular health: what works? Am J Health Promot. 2005;19(3):167-93.

26. Minatto G, Barbosa Filho VC, Berria J, Petroski EL. Schoolbased interventions to improve cardiorespiratory fitness in adolescents: systematic review with meta-analysis. Sports Med. 2016;46(9):1273-92. 
27. Morgan PJ, Barnett LM, Cliff DP, Okely AD, Scott HA, Cohen KE, et al. Fundamental movement skill interventions in youth: a systematic review and meta-analysis. Pediatrics. 2013;132(5):e1361-83.

28. Opstoel K, Chapelle L, Prins FJ, De Meester A, Haerens $\mathrm{L}$, van Tartwijk J, et al. Personal and social development in physical education and sports: A review study. Eur. Phys. Educ. Rev. 2019;1356336X1988205.

29. Quitério ALD. School physical education: The effectiveness of health-related interventions and recommendations for health-promotion practice. Health Educ. J. 2013;72(6):71632.

30. Rasberry CN, Lee SM, Robin L, Laris BA, Russell LA, Coyle KK, et al. The association between school-based physical activity, including physical education, and academic performance: A systematic review of the literature. Prev Med. 2011;52:S10-20.

31. Robinson DB, Randall L, Gleddie DL, Barrett J, Berg S. Canada's 150-minute 'standard' in physical education: a consideration of research evidence related to physical education instructional time. Curriculum Studies in Health and Physical Education. 2019;10(3):226-46.

32. Salmon J, Booth ML, Phongsavan P, Murphy N, Timperio A. Promoting physical activity participation among children and adolescents. Epidemiologic Reviews. 2007;29(1):144-59.

33. Sharma M. School-based interventions for childhood and adolescent obesity. Obes Rev. agosto de 2006;7(3):261-9.

34. Schieffer TM, Thomas KT. Fifteen years of promise in school-based physical activity interventions: a meta-analysis. Kinesiol Rev. 2012;1(3):155-69.

35. Schüller I, Demetriou Y. Physical activity interventions promoting social competence at school: A systematic review. Educ. Res. Rev. 2018;25:39-55.

36. Slingerland M, Borghouts L. Direct and indirect influence of physical education-based interventions on physical activity: a review. J Phys Act Health. 2011;8(6):866-78.

37. Soriano-Férriz, Beatriz. Programas y ejercicios de flexibilidad dentro de las clases de educación física, en niños y niñas escolares, y su efecto en la mejora de la extensibilidad isquiosural: una revisión sistemática. MHSalud.2018;15(1):1.

38. Tompsett C, Sanders R, Taylor C, Cobley S. Pedagogical Approaches to and effects of fundamental movement skill interventions on health outcomes: a systematic review. Sports Med. 2017;47(9):1795-819.

39. Trudeau F, Shephard RJ. Physical education, school physical activity, school sports and academic performance. Int J Behav Nutr Phys Act. 2008;5(1):10.

40. van de Kop JH, van Kernebeek WG, Otten RHJ, Toussaint HM,VerhoeffAP.School-based physical activityinterventions in prevocational adolescents: a systematic review and metaanalyses. J. Adolesc. Health. 2019;65(2):185-94.

41. van Sluijs EMF, McMinn AM, Griffin SJ. Effectiveness of interventions to promote physical activity in children and adolescents: systematic review of controlled trials. BMJ. 2007;335(7622):703.

42. Vazou S, Pesce C, Lakes K, Smiley-Oyen A. More than one road leads to Rome: A narrative review and meta-analysis of physical activity intervention effects on cognition in youth. Int J Sport Exerc Psychol. 2019;17(2):153-78.

43. Vella SA, Braithewaite RE, Gardner LA, Spray CM. A systematic review and meta-analysis of implicit theory research in sport, physical activity, and physical education. Int J Sport Exerc Psychol. 2016;9(1):191-214.
44. White RL, Babic MJ, Parker PD, Lubans DR, Astell-Burt T, Lonsdale C. Domain-specific physical activity and mental health: a meta-analysis. Am. J. Prev. Med. 2017;52(5):653-66.

45. Zach S, Shoval E, Lidor R. Physical education and academic achievement-literature review 1997-2015. Journal of Curriculum Studies. 2017;49(5):703-21.

46. Centers for Disease Control and Prevention. The association between school-based physical activity, including physical education, and academic performance [Internet]. 2010. Disponível em: https:/www.cdc.gov/healthyyouth/health_ and_academics/pdf/pa-pe_paper.pdf

47. Centers for Disease Control and Prevention. School Health Guidelines to Promote Healthy Eating and Physical Activity [Internet]. 2011. Disponível em: https://www.cdc. gov/healthyschools/npao/pdf/MMWR-School-HealthGuidelines.pdf

48. Institute of Medicine. Educating the Student Body: Taking Physical Activity and Physical Education to School [Internet]. Washington, D.C.: National Academies Press; 2013 [citado 14 de julho de 2020]. Disponível em: http:// www.nap.edu/catalog/18314

49. Physical Activity Guidelines Advisory Committee Scientific Report_U.S. 2018 Physical Activity Guidelines Advisory Committee Scientific Report [Internet]. 2018. Disponível em: https://health.gov/sites/default/files/2019-09/PAG_ Advisory_Committee_Report.pdf

\section{Material suplementar 2}

\section{Lista de referências incluídas para a questão 2 "Quais} as evidências de contribuiçôes das intervençôes implementadas nas aulas de EF escolar no Brasil para promoção de um estilo de vida ativo e saudável"

1. Cardeal CM, Pereira LA, Silva PF, França NM. Efeito de um programa escolar de estimulação motora sobre desempenho da função executiva e atenção em crianças. Motri. 2013;9(3):44-56.

2. Coledam DHC, Arruda GA de, Oliveira AR de. Efeito crônico do alongamento estático realizado durante o aquecimento sobre a flexibilidade de crianças. Rev Bras Cineantropom Desempenho Hum. 2012;14(3):296-304.

3. Costa CLA, Nobre GC, Nobre FSS, Valentini NC. The effect of a program for motor intervention on motor development of children at social risk in cariri - Ce. Rev Ed Fisica. 2014;25(3):353-64.

4. Cunha CT, Poblacion AP, Colugnati FAB, Taddei JA de AC, Bracco MM. Effect of an educational program on schoolchildren's energy expenditure during physical education classes. MedicalExpress. 2016;3(1).

5. Farias ES, Paula F, Carvalho WRG, Gonçalves EM, Baldin AD, Guerra-Júnior G. Efeito da atividade física programada sobre a composição corporal em escolares adolescentes. J pediatr (Rio J). 2009;85(1):28-34.

6. Farias E dos S, Carvalho WRG de, Gonçalves EM, GuerraJúnior G. Efeito da atividade física programada sobre a aptidão física em escolares adolescentes. Rev Bras Cineantropom Desempenho Hum. 2010;12(2):98-105.

7. Finco MD, Variani P, Reategui EB, Zaro MA. Exergames as a new support tool for physical education classes. In 2013. p. 360-3. Disponível em: https://www.scopus.com/ 
inward/record.uri?eid=2-s2.0-84883302975\&doi $=10.110$ 9\%2fCTS. 2013.6567255\&partnerID $=40 \& \mathrm{md} 5=0238758$ ae44d57135826f95abc1edb35 https://ieeexplore.ieee.org/ document/6567255/

8. Gonzales NG, Azevedo MR, Böhlke CF, Freitas MP, Rombaldi AJ. Projeto eft: implicações pedagógicas e nível de conhecimento sobre saúde. J Phys Educ (Maringá). 2018;29:e2949-e2949.

9. Rossi Filho S, Lopes da Silva C. Super-heróis e educação para o lazer: descrição de uma proposta pedagógica nas aulas de Educação Física. / Superheroes and education for leisure: description of a pedagogical proposal in Physical Education classes. Revista Brasileira de Ciência e Movimento: RBCM. 2019;27(2):188-208.

10. Silva AZ da, Pereira FLH, Mincewicz G, Araujo LB de, Guimarães ATB, Israel VL. Psychomotor Intervention to stimulate Motor Development in 8-10-year-old schoolchildren. Rev Bras Cineantropom Desempenho Hum. 2017;19(2):150-63.

11. Silveira SR, Basso L, Freudenheim AM, Corrêa UC, Ferreira MG, Tani G. Aquisição da habilidade motora rebater na educação física escolar: um estudo das dicas de aprendizagem como conteúdo de ensino. Rev Bras Educ Fís Esporte. 2013;27(1):149-57.

12. Spohr C, Fortes M, Rombaldi A, Hallal P, Azevedo M. Atividade física e saúde na Educação Física escolar: efetividade de um ano do projeto "Educação Física +". Rev Bras Ativ Fís Saúde. 2014;19(3):300-13.

13. Oliveira L, Braga F, Lemes V, Dias A, Brand C, Mello J, et al. Effect of an intervention in Physical Education classes on health related levels of physical fitness in youth. Rev Bras Ativ Fís Saúde 2017;22(1):46-53.

14. Zanetti MC, Feltran GN, Polito LFT, Dias HM, Neves AN, Brandão MRF. An intervention program on motivation and psychological needs in physical education. Rev Psicol Del Deporte. 2017;26:34-8.

15. Barbosa Filho VC, Lopes Ada S, Lima AB, de Souza EA, Gubert Fdo A, Silva KS, et al. Rationale and methods of a cluster-randomized controlled trial to promote active and healthy lifestyles among Brazilian students: the "Fortaleça sua Saúde" program. BMC public health. 2015;15:1212.

16. Barbosa Filho VC, da Silva KS, Mota J, Beck C, Lopes A da $\mathrm{S}$. A physical activity intervention for Brazilian students from low human development index areas: A cluster-randomized controlled trial. J PhysAct Health. 2016;13(11):1174-82.

17. Barbosa Filho VC, da Silva KS, Mota J, Vieira NFC, Gubert FDA, Lopes ADS. "For whom was it effective?" Moderators of the effect of a school-based intervention on potential physical activity determinants among Brazilian students. Prev Med. 2017;97:80-5.

18. Barbosa Filho VC, Bandeira ADS, Minatto G. Effect of a multicomponent intervention on lifestyle factors among brazilian adolescents from low human development index areas: a cluster-randomized controlled trial. Int. J. Environ. Res. Public Health 2019, 16, 267

19. Bandeira ADS, Silva KS, Bastos JLD, Silva DAS, Lopes ADS, Barbosa Filho VC. Psychosocial mediators of screen time reduction after an intervention for students from schools in vulnerable areas: A cluster-randomized controlled trial. Journal of science and medicine in sport. 2020;23(3):264-9.

20. Lopes IE, Linard JG, da Silva ML, Filho VCB. Implementação do programa de promoção do estilo de vida ativo em estudantes: O "Fortaleça sua saúde". Journal of Physical Education (Maringa) [Internet]. 2020;31(1).
21. Bandeira A, da Silva KS, de Sá SAM, Guerra PH, Mota J, Barbosa Filho VC. Effect of a multicomponent intervention on variables related to screen time in adolescents: a clusterrandomized controlled trial. Rev Bras Ativ Fís Saúde. 2018;23:1-8.

22. Nahas MV, de Barros MV, de Assis MA, Hallal PC, Florindo AA, Konrad L. Methods and participant characteristics of a randomized intervention to promote physical activity and healthy eating among brazilian high school students: the Saude na Boa project. J Phys Act Health. 2009;6(2):153-62.

23. Barros MVG, Nahas MV, Hallal PC, de Farias Júnior JC, Florindo AA, de Barros SSH. Effectiveness of a SchoolBased Intervention on Physical Activity for High School Students in Brazil: The Saude na Boa Project. J Phys Act Health. 2009;6(2):163-9.

24. Del Duca GF, Gomes de Barros MV, Samara Silva K, Totaro Garcia LM, Bezerra J, Vinicius Nahas M. Effectiveness on physical activity indicators of an intervention delivered to high school students. Rev Bras Cineantropom Desempenho Hum. 2014;16:13-24.

25. Costa FF, Altenburg de Assis MA, González-Chica D, Bernardo C, Gomes de Barros MV, Vinicius Nahas M. Effect of school-based intervention on diet in high school students. Rev Bras Cineantropom Desempenho Hum. 2014;16:37-45.

26. Ferreira de Sousa T, Vinicius Nahas M, Menêses Hardman C, Totaro Garcia LM, Del Duca GF, Altenburg de Assis MA. Effect of intervention on total and central obesity in students: The Saúde na Boa project. Rev Bras Cineantropom Desempenho Hum. 2014;16:47-54.

27. Hardman CM, Barros MVG de, Lopes AS, Lima RA, Bezerra J, Nahas MV. Efetividade de uma intervenção de base escolar sobre o tempo de tela em estudantes do ensino médio. Rev Bras Cineantropom Desempenho Hum. 2014;16(0):25.

28. Silva KS, Barros MVG de, Barbosa Filho VC, Garcia LMT, Wanderley Júnior R de S, Beck CC, et al. Implementation of the "Saúde na Boa" intervention: process evaluation and characteristics of participants and dropouts. Rev Bras Cineantropom Desempenho Hum. 2014;16:01-12.

29. Knebel MTG, Borgatto AF, Lopes MVV, dos Santos PC, Matias TS, Narciso FV, et al. Mediating role of screen media use on adolescents' total sleep time: A cluster-randomized controlled trial for physical activity and sedentary behaviour. Child: Care, Health and Development. 2020;46(3):381-9.

30. Medeiros PFP, Cogo-Moreira H, Leme AC, Philippi ST.The "Healthy Habits, Healthy Girls" randomized controlled trial for girls: study design, protocol, and baseline results. $\mathrm{PloS}$ one Cad Saude Publica. 2015;31(7):1381-94.

31. Leme AC, Lubans DR, Guerra PH, Dewar D, Toassa EC, Philippi ST. Preventing obesity among Brazilian adolescent girls: Six-month outcomes of the Healthy Habits, Healthy Girls-Brazil school-based randomized controlled trial. Preventive medicine. 2016;86:77-83.

32. Leme ACB, Philippi ST, Thompson D, Nicklas T, Baranowski T. "Healthy Habits, Healthy Girls-Brazil": an obesity prevention program with added focus on eating disorders. Eating and weight disorders: EWD. 2019;24(1):107-19.

33. Minatto G, Petroski EL, Da Silva KS, Duncan MJ. Schoolbased intervention on cardiorespiratory fitness in Brazilian students: A nonrandomized controlled trial. J. Funct. Morphol. Kinesiol. 2019;4(1),10.

34. Vieira JAJ, Lima LRA de, Silva DAS, Petroski EL. Effectiveness of a multicomponent intervention on the screen time of Brazilian adolescents: non-randomized controlled study. Motriz: rev educ fis [Internet]. 2018;24(3). 
35. Costa BGG da, Silva KS da, Silveira PM, Berria J, Machado AR, Petroski EL. Efeito de uma intervenção sobre atividade física moderada a vigorosa e comportamento sedentário no tempo escolar de adolescentes. Rev bras epidemiol [Internet]. 2019;22:E190065.

\section{Material suplementar 3}

\section{Lista de documentos incluídos para a questão 3} "Quais as estratégias relacionadas às aulas de EF escolar têm sido recomendadas para promoção de um estilo de vida ativo e saudável em escolares no mundo?"

1. Bogden JF. Fit, Healthy, and Ready To Learn: A School Health Policy Guide. Part I: Physical Activity, Health Eating, and Tobacco-Use Prevention. março de 2000;1-230.

2. Federation Internationale d'Éducation Physique. The World Manifest of Physical Education FIEP 2000 [Internet]. 2000 [citado 5 de julho de 2020]. Disponível em: http://fiepeurope. eu/manifest.php

3. Task Force on Community Preventive Services. Increasing physical activity. A report on recommendations of the Task Force on Community Preventive Services. MMWR Recommendations and reports: Morbidity and mortality weekly report Recommendations and reports. 2001/11/09 ed 26 de outubro de 2001;50( $\operatorname{Rr}-18): 1-14$.

4. Haney L. Physical education statement by the President's Council on Physical Fitness and Sports. NASNewsletter. 2002/05/04 ed janeiro de 2001;16(1):13.

5. Barrios LC, Sleet DA, Mercy JA. CDC School Health Guidelines to Prevent Unintentional Injuries and Violence. American Journal of Health Education [Internet]. 2003 [citado 17 de junho de 2020];34(5). Disponível em: https:// eric.ed.gov/:id=EJ853628

6. Hayman LL, Williams CL, Daniels SR, Steinberger J, Paridon S, Dennison BA, et al. Cardiovascular health promotion in the schools: a statement for health and education professionals and child health advocates from the Committee on Atherosclerosis, Hypertension, and Obesity in Youth (AHOY) of the Council on Cardiovascular Disease in the Young, American Heart Association. Circulation. 2004/10/13 ed 12 de outubro de 2004;110(15):2266-75.

7. American Academy of Pediatrics. Active healthy living: prevention of childhood obesity through increased physical activity. Pediatrics. 2006/05/03 ed maio de 2006;117(5):1834-42.

8. Pate RR, Davis MG, Robinson TN, Stone EJ, McKenzie TL, Young JC. Promoting physical activity in children and youth: A leadership role for schools - A scientific statement from the American Heart Association Council on Nutrition, Physical Activity, and Metabolism (Physical Activity Committee) in collaboration with the Councils on Cardiovascular Disease in the Young and Cardiovascular Nursing. Circulation. 2006;114(11):1214-24.

9. Centers for Disease Control and Prevention. A CDC Review of School Laws and Policies Concerning Child and Adolescent Health. Journal of School Health. 2008;78(2):69-128.

10. National Association for Sport and Physical Education. A Position Statement from the National Association for Sport and Physical Education: Comprehensive School Physical Activity Program. Strategies: A Journal for Physical and Sport Educators. 2008;21(6):29-33.

11. Pate RR, O'Neill JR. Summary of the American Heart Association scientific statement: promoting physical activity in children and youth: a leadership role for schools. J Cardiovasc. Nurs. 2008;23(1):44-9.
12. Mandigo J, Francis PDN, Lodewyk EDK. Position Paper Physical Literacy for Educators. 2009;13.

13. Ministerio de la Protección Social. Guía para el desarrollo de programas intersectoriales y comunitarios para la promoción de la actividad física: programa nacional de actividad física Colombia activa y saludable [Internet]. Imprenta Nacional de Colombia; 2009. Disponível em: https://books.google.com. br/books?id=MWiAswEACAAJ

14. SHAPE America. Appropriate Instructional Practice Guidelines, K-12: A Side-by-Side Comparison SHAPE America - Society of Health and Physical Educators [Internet]. 2009 [citado 5 de julho de 2020]. Disponível em: https://www.shapeamerica.org/upload/AppropriateInstructional-Practice-Guidelines-K-12.pdf

15. Ministry of Social Affairs and Health. Recommendations for the promotion of physical activity in Finland. 2010.

16. International Council of Sport Science and Physical EducationScience Education Policy. International Position Statement on Physical Education. 2010.

17. SHAPE America. Opportunity to Learn: Guidelines for Elementary, Middle \& High School Physical Education - A Side-by-Side Comparison [Internet]. 2010 [citado 5 de julho de 2020]. Disponível em: https://www.shapeamerica.org/ standards/guidelines/upload/Opportunity-to-Learn-Grid.pdf

18. Centers for Disease Control and Prevention. The Association Between School-Based Physical Activity, Including Physical Education, and Academic Performance. 2010.

19. Edginton CR, Chin MK, Geadelmann PL, AhrabiFard I. Global Forum for Physical Education Pedagogy 2010 (GoFPEP 2010): Health and Physical Education Pedagogy in the 21st Century - A Statement of Consensus. International Journal of Physical Education. 2nd Quarter de 2011;48(2):33-41.

20. Costa Rica. Ministerio de Salud y Ministerio de Deporte y Recreación. Plan Nacional de Actividad Física y Salud 20112021. 2011.

21. Ecuador. Ministerio de salud pública Del Ecuador, Coordinación Nacional de Nutrición. Guia de Actividad Física dirigida al personal de salud II. 2011.

22. Centers for Disease Control and Prevention. School Health Guidelines to Promote Healthy Eating and Physical Activity: Executive Summary. setembro de 2011;1-8.

23. Ministerio de Salud Pública y Bienestar Social. Directrices de Evaluación de Niñas, Niños y Adolescentes para la Actividad Física Pedagógica Recreativa y Deportiva Escolar en Paraguay. Pediatr (Asunción). abril de 2012;39(1):47-56.

24. ParticipACTION Advisory Groups. Active Canada 20/20: A physical activity strategy and change agenda for Canada. 2012.

25. Centers for Disease Control and Prevention. Parent Engagement: Strategies for Involving Parents in School Health. 2012.

26. International Council of Sport Science and Physical EducationScience Education Policy. International Benchmarks for Physical Education Systems Developed by ICSSPE's International Committee of Sport Pedagogy. 2012.

27. SHAPE America. Instructional Framework for Fitness Education In Physical Education [Internet]. 2012 [citado 5 de julho de 2020]. Disponível em: https://www.shapeamerica. org/standards/guidelines/upload/Instructional-Frameworkfor-Fitness-Education-in-Physical-Education.pdf

28. Centers for Disease Control and Prevention. Promoting Parent Engagement: Improving Student Health and Academic Achievement. 2012. 
29. Incarbone O, Ferrante D, Bazan N, Gonzalez G, Barengo N, Kanfino J. Manual Director de actividad física y salud de la república Argentina. Plan Nacional Argentina Saludable Dirección de Promoción de la Salud y Control de Enfermedades No Transmisibles Ministerio de Salud de la Nación. 2013;8(9).

30. Kay T, Dudfield O, Commonwealth Secretariat. The commonwealth guide to advancing development through sport. London: Commonwealth Secretariat; 2013.

31. UNESCO. Declaration of Berlin: International Conference of Ministers and Senior Officials Responsible for Physical Education and Sport [Internet]. 2013 [citado 5 de julho de 2020]. Disponível em: https://unesdoc.unesco.org/ ark:/48223/pf0000221114

32. Oliveira ARC, Sartori SK, Laurindo E. Recomendações para a Educação Física escolar. Sistema CONFEF/CREFs Conselhos Federal e Regionais de Educação Física. 2014;

33. Lloyd RS, Faigenbaum AD, Stone MH, Oliver JL, Jeffreys I, Moody JA, et al. Position statement on youth resistance training: the 2014 International Consensus. British Journal of Sports Medicine. 2014;48(7):498-505.

34. Graf C, Beneke R, Bloch W, Bucksch J, Dordel S, Eiser S, et al. Recommendations for promoting physical activity for children and adolescents in Germany. A consensus statement. Obesity Facts. 2014;7(3):178-90.

35. Buscemi J, Kong A, Fitzgibbon ML, Bustamante EE, Davis CL, Pate RR, et al. Society of Behavioral Medicine position statement: elementary school-based physical activity supports academic achievement. Translational behavioral medicine. 2014;4(4):436-8.

36. SHAPE America. The Essential Components of Physical Education [Internet]. 2015 [citado 5 de julho de 2020]. Disponível em: https://www.shapeamerica.org/upload/ TheEssentialComponentsOfPhysicalEducation.pdf

37. UNESCO. International Charter of Physical Education, Physical Activity and Sport [Internet]. 2015 [citado 5 de julho de 2020]. Disponível em: https://unesdoc.unesco.org/ ark:/48223/pf0000235409

38. UNESCO. Diretrizes em educação física de qualidade (EFQ) para gestores de políticas [Internet]. 2015 [citado 5 de julho de 2020]. Disponível em: https://unesdoc.unesco. org/ark:/48223/pf0000231963

39. The common Wealth. Sport for Development and Peace and the 2030 Agenda for Sustainable Development [Internet]. 2015 [citado 5 de julho de 2020]. Disponível em: https:// thecommonwealth.org/sites/default/files/inline/CW SDP_2030\%2BAgenda.pdf

40. American Heart Association. Increasing and Improving Physical Education and Physical Activity in Schools: Benefits for Children's Health and Educational Outcomes [Internet]. 2015 [citado 5 de julho de 2020]. Disponível em: https://www.heart.org/idc/groups/heart-public/@wcm/@ adv/documents/downloadable/ucm_473782.pdf

41. Ministerio del Deporte, Gobierno de Chile. Política Nacional de Actividad Física y Deporte 2016-2025 [Internet]. 2016 [citado 2 de julho de 2020]. Disponível em: http://www. mindep.cl/wp-content/uploads/2015/05/POLITICAULTIMA-VERSI\%C3\%93N-021116.pdf

42. Rütten A, Pfeifer K, Banzer W, Ferrari N, Füzéki E, Geidl W, et al. National Recommendations for Physical Activity and Physical Activity Promotion [Internet]. 2016 [citado 3 de julho de 2020]. Disponível em: https://opus4.kobv.de/ opus4-fau/frontdoor/index/index/docId/7827
43. SHAPE America. Guide for the Physical Education Policy [Internet]. 2016 [citado 5 de julho de 2020]. Disponível em: https://www.shapeamerica.org/advocacy/upload/Guide-forPhysical-Education-Policy-9-23-14.pdf

44. SHAPE America. Shape of the Nation Status of Physical Education in the USA [Internet]. 2016 [citado 5 de julho de 2020]. Disponível em: https://www.shapeamerica.org/ advocacy/son/2016/upload/Shape-of-the-Nation-2016_ web.pdf

45. Department of Health (Ireland). Get Ireland Active! The National Physical Activity Plan for Ireland [Internet]. 2016 [citado 5 de julho de 2020]. Disponível em: https://assets. gov.ie/12198/5f3dbab207f2464bba3b9b3f6d02bff6.pdf

46. National Physical Activity Plan Alliance. National Physical Activity Plan Alliance. U.S. National Physical Activity Plan. 2016 [Internet]. 2016 [citado 5 de julho de 2020]. Disponível em: https://www.physicalactivityplan.org/docs/2016NPAP_ Finalforwebsite.pdf

47. The Australian Council for Health, Physical Education and Recreation (ACHPER). ACHPER NATIONAL POSITION STATEMENT: Support of the Australian Curriculum: Health and Physical Education [Internet]. 2017 [citado 13 de julho de 2020]. Disponível em: https:/www. achper.org.au/documents/item/393

48. The common Wealth. Enhancing the Contribution of Sport to the Sustainable Development Goals [Internet]. OECD Publishing; 2017 [citado 5 de julho de 2020]. Disponível em: http://www.thecommonwealth-ilibrary.org/commonwealth/ development/enhancing-the-contribution-of-sport-to-thesustainable-development-goals_9781848599598-en

49. Centers for Disease Control and Prevention. School Health Index: A Self-Assessment and Planning Guide Elementary School 2017 [Internet]. 2017 [citado 5 de julho de 2020]. Disponível em: https:/www.cdc.gov/healthyschools/shi/pdf/ Elementary-Total-2017.pdf

50. Centers for Disease Control and Prevention. School Health Index: A Self-Assessment and Planning Guide Middle and High School 2017 [Internet]. 2017 [citado 5 de julho de 2020]. Disponível em: https://www.cdc.gov/healthyschools/ shi/pdf/Middle-High-Total-2017.pdf

51. SHAPE America. Physical Education is Essential for All Students: No Substitutions, Waivers or Exemptions for Physical Education. 2018;6.

52. World Health Organization. Global action plan on physical activity 2018-2030: more active people for a healthier world [Internet]. 2018 [citado 5 de julho de 2020]. Disponível em: https://www.who.int/ncds/prevention/physical-activity/ global-action-plan-2018-2030/en/

53. European Physical Education Association. European Framework of Quality Physical Education [Internet]. 2018 [citado 5 de julho de 2020]. Disponível em: http:// www.eupea.com/wp-content/uploads/2018/02/EuropeanFramework-of-Quality-PE.pdf

54. US Department of Health and Human Services. Physical activity guidelines advisory committee scientific report. Washington (DC): US Department of Health and Human Services. 2018;

55. Departament of Health of Australia. Australian 24-Hour Movement Guidelines for Children (5-12 years) and Young People (13-17 years): An Integration of Physical Activity, Sedentary Behaviour, and Sleep. 2019.

56. Centers for Disease Control and Prevention. Parents for Healthy Schools: A Guide for Getting Parents Involved from K-12. 2019;26. 
57. Organisation for Economic Co-operation and Development (OECD). Making Physical Education Dynamic and Inclusive for 2030: International Curriculum Analysis [Internet]. 2019 [citado 5 de julho de 2020]. Disponível em: https://www.oecd. org/education/2030-project/contact/OECD_FUTURE_ OF_EDUCATION_2030_MAKING_PHYSICAL DYNAMIC_AND_INCLUSIVE_FOR_2030.pdf

58. Association for Physical Education (afPE). The Inspection and Maintenance of Gymnastics, Sports Hall, Fixed Play, Fitness and Sports Equipment [Internet]. 2019 [citado 5 de julho de 2020]. Disponível em: https://www.afpe.org. uk/physical-education/wp-content/uploads/Inspection-ofEquipment-of-PESSPA-Web.pdf

59. SHAPE America. Getting to Know Your Child's PE Program: A Parent's Guide [Internet]. 2019 [citado 5 de julho de 2020]. Disponível em: https://www.shapeamerica.org/ uploads/pdfs/2017/downloads/eguides/Parent_Checklist.pdf

60. The common Wealth. Measuring the contribution of sport, physical education and physical activity to the Sustainable Development Goals Toolkit and model indicators [Internet]. 2019 [citado 5 de julho de 2020]. Disponível em: https:// thecommonwealth.org/sites/default/files/inline/SportSDGs-Indicator-Framework.pdf
61. The common Wealth. Model indicators on sport, physical education and physical activity and the Sustainable Development Goals [Internet]. 2019 [citado 5 de julho de 2020]. Disponível em: https://thecommonwealth. org/sites/default/files/inline/Sport\%20and\%20SDG\%20 Indicators\%20v3.1.pdf

62. Association for Supervision and Curriculum Development (ASCD). ASCD's Position on the Whole Child [Internet]. 2020 [citado 5 de julho de 2020]. Disponível em: http://files. ascd.org/pdfs/programs/WholeChildNetwork/2020-wholechild-network-learning-compact-renewed.pdf

63. Association for Physical Education. Health Position Paper afPE 2020 [Internet]. 2020 [citado 5 de julho de 2020]. Disponível em: https://www.afpe.org.uk/physical-education/ wp-content/uploads/Health-Position-Paper-2020-Web.pdf 